\title{
Decentralized Multi-objective Bilevel Decision Making with Fuzzy Demands
}

\author{
Guangquan Zhang, Jie Lu and Tharam Dillon \\ Faculty of Information Technology, University of Technology, Sydney \\ PO Box 123, Broadway, NSW 2007, Australia
}

\begin{abstract}
In a bilevel decision problem, the leader at the upper level attempts to optimize his/her objective, the follower at the lower level tries to find an optimized strategy according to each of possible decisions made by the leader. A bilevel decision problem may involve fuzzy demands which appear either in the objective functions or constraints of the leader or the follower or both. Furthermore, the leader and the follower may have multiple conflict objectives that should be optimized simultaneously. This study addresses both issues to propose a fuzzy multi-objective bilevel programming model. It then develops an approximation branch-and-bound algorithm to solve fuzzy multi-objective bilevel decision problems. Finally, two case based examples further illustrate the proposed model and algorithm.
\end{abstract}

Key words: Bilevel programming, Branch-and-bound algorithm, Fuzzy sets, Optimization, Multi-objective decision making, Fuzzy bilevel programming

\section{Introduction}

Decision making in management has often involved two levels of decision makers, uncertain information, and multiple conflicting objectives. With the complex decision making environment, knowledge based intelligent systems, including fuzzy sets and logic, neural networks, optimization algorithms, etc, provide effective assistant for decision problem recognition, modeling and solving.

Bilevel programming (BP) arises where decisions are made in a two level hierarchical order and each decision maker has no direct control upon the

Email address: $\{$ zhangg, jielu, tharam\}@it.uts.edu.au (Guangquan Zhang, Jie Lu and Tharam Dillon).

Preprint submitted to Knowledge-Based System 3 January 2007 
decision of the other, but actions taken by one decision maker effect returns from the other $[1-8,10,26,27]$. Decision maker at the upper level is termed as the leader, and at the lower level, the follower. The leader and the follower each tries to optimize his/her own objective function(s), but the decision affects the objective values of the other level [12]. As decision environments become more complex, two issues below need to be considered when model a real-world bilevel decision problem and find a solution for the problem. This paper will address both issues.

First, the upper level or the lower level or both of a bilevel decision have multiple conflicting objectives which should be considered simultaneously by the decision makers. For example, a coordinator of a multi-division firm considers three objectives in making an aggregate production plan: maximize net profits, maximize quality of products, and maximize worker satisfaction. The three objectives are in conflict with each other, but must be considered simultaneously by the coordinators. Any improvement in one objective may be achieved only at the expense of others. One level multi-objective decision-making problem has been well researched by other researchers such as Hwang \& Masud [9]. But in a bilevel model, the selection of a solution for the leader is affected by his/her followers' optimal reactions. Therefore, to find a solution for the leader which has multiple objectives needs to consider both the compromised solution of the leader and his/her follower's decision.

Second, decision makers have fuzzy demands. This is expressed as that the parameters of the objective functions and the constraints of the leader and the followers cannot be described by precise values. In the problem-formulation process, the parameters are required to be obtained through some experiments and/or some experts' understanding of the nature of the parameters. It has been observed that, in most real-world situations, for example, power market and business management, the possible values of these parameters are often only imprecisely or ambiguously known to the experts. With this observation, it would be certainly more appropriate to interpret the experts' understanding of the parameters as fuzzy numerical data which can be represented by means of fuzzy sets [28].

Research on fuzzy BP have been reported in literatures. For example, Sakawa et al. [14-21] formulated cooperative fuzzy BP problems and proposed an interactive fuzzy programming approach for solving the problem. In their approach, the concepts of $\alpha$-bilevel programming was introduced based on the basis of fuzzy number $\alpha$-level sets. At the same time, some researches applied fuzzy set technique to deal with BP problems. For example, Shih [24] applied fuzzy set theory to overcome the computational difficulties in solving bilevel problems, and Shina [25] applied fuzzy mathematical programming approach to obtain the solution of multi-level linear programming problems. 
Our research applies fuzzy sets to deal with fuzzy linear BP (FLBP) problem when the leader or the follower or both have multiple objectives. Based on the extended solution concept and related theorems of BP [11,22,23], we have first solved FLBP problems with a specialized forms of membership functions, triangular form, in the fuzzy parameters [29-31] and then in the general form of fuzzy numbers [32]. This paper extends our previous research by allowing the leader and the follower to have multiple objectives with fuzzy parameters, called a fuzzy multi-objective linear bilevel programming (FMOLBP) problem. This paper in particular develops an approximation branch-and-bound algorithm to solve the FMOLBP problems.

Following the introduction, Section 2 gives some basic concepts and theorems regarding to FMOLBP problems. Section 3 presents a FMOLBP model, related definitions, theorems and properties. A general fuzzy number based approximation branch-and-bound algorithm for solving FMOLBP problems are proposed in Section 4. Two cased based examples are shown in Section 5 for illustrating the proposed model and algorithm. Conclusions and further study are discussed in Section 6.

\section{Preliminaries}

In this section, we present some basic concepts, definitions and theorems that are to be used in the subsequent sections. The work presented in this section can also be found from our recent papers in $[31,32]$.

Let $\mathbb{R}$ be the set of all real numbers, $\mathbb{R}^{n}$ be $n$-dimensional Euclidean space, and $x=\left(x_{1}, x_{2}, \cdots, x_{n}\right)^{T}, y=\left(y_{1}, y_{2}, \cdots, y_{n}\right)^{T} \in \mathbb{R}^{n}$ be any two vectors, where $x_{i}, y_{i} \in \mathbb{R}, i=1,2, \ldots, n$ and $T$ denotes the transpose of the vector. Then we denote the inner product of $x$ and $y$ by $\langle x, y\rangle$. For any two vectors $x, y \in \mathbb{R}^{n}$, we write

$$
\begin{aligned}
& x \geqq y \text { iff } x_{i} \geqslant y_{i}, \forall i=1,2, \ldots, n ; \\
& x \geq y \text { iff } x \geqq y \text { and } x \neq y ; \\
& x>y \text { iff } x_{i}>y_{i}, \forall i=1,2, \ldots, n .
\end{aligned}
$$

Definition 1 A fuzzy number $\tilde{a}$ is defined as a fuzzy set on $\mathbb{R}$, whose membership function $\mu_{\tilde{a}}$ satisfies the following conditions:

(1) $\mu_{\tilde{a}}$ is a mapping from $\mathbb{R}$ to the closed interval $[0,1]$;

(2) it is normal, i.e., there exists $x \in \mathbb{R}$ such that $\mu_{\tilde{a}}(x)=1$;

(3) for any $\lambda \in(0,1], a_{\lambda}=\left\{x ; \mu_{\tilde{a}}(x) \geqslant \lambda\right\}$ is a closed interval, denoted by $\left[a_{\lambda}^{L}, a_{\lambda}^{R}\right]$ 
Let $\mathscr{F}(\mathbb{R})$ be the set of all fuzzy numbers. By the decomposition theorem of fuzzy sets, we have

$$
\tilde{a}=\bigcup_{\lambda \in[0,1]} \lambda\left[a_{\lambda}^{L}, a_{\lambda}^{R}\right]
$$

for every $\tilde{a} \in \mathscr{F}(\mathbb{R})$.

Let $\mathscr{F}^{*}(\mathbb{R})$ be the set of all finite fuzzy numbers on $\mathbb{R}$.

Theorem 2 Let $\tilde{a}$ be a fuzzy set on $\mathbb{R}$, then $\tilde{a} \in \mathscr{F}(\mathbb{R})$ if and only if $\mu_{\tilde{a}}$ satisfies

$$
\mu_{\tilde{a}}(x)=\left\{\begin{array}{l}
1, \quad x \in[m, n] \\
L(x), \quad x<m \\
R(x), x>n
\end{array}\right.
$$

where $L(x)$ is the right-continuous monotone increasing function, $0 \leqslant L(x)<$ 1 and $\lim _{x \rightarrow-\infty} L(x)=0, R(x)$ is the left-continuous monotone decreasing function, $0 \leqslant R(x)<1$ and $\lim _{x \rightarrow \infty} R(x)=0$.

Corollary 3 For every $\tilde{a} \in \mathscr{F}(\mathbb{R})$ and $\lambda_{1}, \lambda_{2} \in[0,1]$, if $\lambda_{1} \leqslant \lambda_{2}$, then $a_{\lambda_{2}} \subset$ $a_{\lambda_{1}}$.

Definition 4 For any $\tilde{a}, \tilde{b} \in \mathscr{F}(\mathbb{R})$ and $0 \leqslant \lambda \in \mathbb{R}$, the sum of $\tilde{a}$ and $\tilde{b}$ and the scalar product of $\lambda$ and $\tilde{a}$ are defined by the membership functions

$$
\begin{aligned}
\mu_{\tilde{a}+\tilde{b}}(t) & =\sup \min _{t=u+v}\left\{\mu_{\tilde{a}}(u), \mu_{\tilde{b}}(v)\right\}, \\
\mu_{\tilde{a}-\tilde{b}}(t) & =\sup \min _{t=u-v}\left\{\mu_{\tilde{a}}(u), \mu_{\tilde{b}}(v)\right\}, \\
\mu_{\lambda \tilde{a}}(t) & =\sup _{t=\lambda u} \mu_{\tilde{a}}(u) .
\end{aligned}
$$

Theorem 5 For any $\tilde{a}, \tilde{b} \in \mathscr{F}(\mathbb{R})$ and $0 \leqslant \alpha \in \mathbb{R}$,

$$
\begin{aligned}
\tilde{a}+\tilde{b} & =\bigcup_{\lambda \in[0,1]} \lambda\left[a_{\lambda}^{L}+b_{\lambda}^{L}, a_{\lambda}^{R}+b_{\lambda}^{R}\right], \\
\tilde{a}-\tilde{b} & =\tilde{a}+(-\tilde{b})=\bigcup_{\lambda \in[0,1]} \lambda\left[a_{\lambda}^{L}-b_{\lambda}^{R}, a_{\lambda}^{R}-b_{\lambda}^{L}\right], \\
\alpha \tilde{a} & =\bigcup_{\lambda \in[0,1]} \lambda\left[\alpha a_{\lambda}^{L}, \alpha a_{\lambda}^{R}\right] .
\end{aligned}
$$

Definition 6 Let $\tilde{a}_{i} \in \mathscr{F}(\mathbb{R}), i=1,2, \cdots, n$. We define $\tilde{a}=\left(\tilde{a}_{1}, \tilde{a}_{2}, \cdots, \tilde{a}_{n}\right)$

$$
\begin{aligned}
\mu_{\tilde{a}}: \mathbb{R}^{n} & \rightarrow[0,1] \\
x & \mapsto \bigwedge_{i=1}^{n} \mu_{\tilde{a}_{i}}\left(x_{i}\right),
\end{aligned}
$$

where $x=\left(x_{1}, x_{2}, \ldots, x_{n}\right)^{T} \in \mathbb{R}^{n}$, and $\tilde{a}$ is called an $n$-dimensional fuzzy 
number on $\mathbb{R}^{n}$. If $\tilde{a}_{i} \in \mathscr{F}^{*}(\mathbb{R}), i=1,2, \ldots, n, \tilde{a}$ is called an $n$-dimensional finite fuzzy number on $\mathbb{R}^{n}$.

Let $\mathscr{F}\left(\mathbb{R}^{n}\right)$ and $\mathscr{F}^{*}\left(\mathbb{R}^{n}\right)$ be the set of all $n$-dimensional fuzzy numbers and the set of all $n$-dimensional finite fuzzy numbers on $\mathbb{R}^{n}$ respectively.

Proposition 7 For every $\tilde{a} \in \mathscr{F}\left(\mathbb{R}^{n}\right)$, $\tilde{a}$ is normal.

Proposition 8 For every $\tilde{a} \in \mathscr{F}\left(\mathbb{R}^{n}\right)$, the $\lambda$-section of $\tilde{a}$ is an $n$-dimensional closed rectangular region for any $\lambda \in[0,1]$.

Proposition 9 For every $\tilde{a} \in \mathscr{F}\left(\mathbb{R}^{n}\right)$ and $\lambda_{1}, \lambda_{2} \in[0,1]$, if $\lambda_{1} \leqslant \lambda_{2}$, then $a_{\lambda_{2}} \subset a_{\lambda_{1}}$.

Definition 10 For any $n$-dimensional fuzzy numbers $\tilde{a}, \tilde{b} \in \mathscr{F}\left(\mathbb{R}^{n}\right)$, we define

(1) $\tilde{a} \succsim \tilde{b}$ iff $a_{\lambda}^{L} \geqq b_{\lambda}^{L}$ and $a_{\lambda}^{R} \geqq b_{\lambda}^{R}, \lambda \in(0,1]$;

(2) $\tilde{a} \succsim \tilde{b}$ iff $a_{\lambda}^{L} \geq b_{\lambda}^{L}$ and $a_{\lambda}^{R} \geq b_{\lambda}^{R}, \lambda \in(0,1]$;

(3) $\tilde{a} \succ \tilde{b}$ iff $a_{\lambda}^{L}>b_{\lambda}^{L}$ and $a_{\lambda}^{R}>b_{\lambda}^{R}, \lambda \in(0,1]$.

We call the binary relations $\succsim, \succsim$ and $\succ$ a fuzzy max order, a strict fuzzy max order and a strong fuzzy max order, respectively.

\section{A Model and Solution Concepts for Fuzzy Multi-Objective Lin- ear Bilevel Programming Model}

Based on our discussion in previous sections, a FMOLBP problem can be modeled as following:

For $x \in X \subset \mathbb{R}^{n}, y \in Y \subset \mathbb{R}^{m}, F: X \times Y \rightarrow \mathscr{F}^{*}\left(\mathbb{R}^{s}\right)$, and $f: X \times Y \rightarrow$ $\mathscr{F}^{*}\left(\mathbb{R}^{t}\right)$

$$
\begin{aligned}
& \min _{x \in X} F(x, y)=\left(\tilde{c}_{11} x+\tilde{d}_{11} y, \tilde{c}_{21} x+\tilde{d}_{21} y, \cdots, \tilde{c}_{s 1} x+\tilde{d}_{s 1} y\right)^{T} \\
& \text { subject to } \tilde{A}_{1} x+\tilde{B}_{1} y \precsim \tilde{b}_{1} \\
& \qquad \min _{y \in Y} f(x, y)=\left(\tilde{c}_{12} x+\tilde{d}_{12} y, \tilde{c}_{22} x+\tilde{d}_{22} y, \cdots, \tilde{c}_{t 2} x+\tilde{d}_{t 2} y\right)^{T} \\
& \quad \text { subject to } \tilde{A}_{2} x+\tilde{B}_{2} y \precsim \tilde{b}_{2}
\end{aligned}
$$

where $\tilde{c}_{i 1}, \tilde{c}_{j 2} \in \mathscr{F}^{*}\left(\mathbb{R}^{n}\right), \tilde{d}_{i 1}, \tilde{d}_{j 2} \in \mathscr{F}^{*}\left(\mathbb{R}^{m}\right), i=1,2, \ldots, s, j=1,2, \ldots, t$, $\tilde{b}_{1} \in \mathscr{F}^{*}\left(\mathbb{R}^{p}\right), \tilde{b}_{2} \in \mathscr{F}^{*}\left(\mathbb{R}^{q}\right), \tilde{A}_{1}=\left(\tilde{a}_{i j}\right)_{p \times n}, \tilde{a}_{i j} \in \mathscr{F}^{*}(\mathbb{R}), \tilde{B}_{1}=\left(\tilde{b}_{i j}\right)_{p \times m}$, $\tilde{b}_{i j} \in \mathscr{F}^{*}(\mathbb{R}), \tilde{A}_{2}=\left(\tilde{e}_{i j}\right)_{q \times n}, \tilde{e}_{i j} \in \mathscr{F}^{*}(\mathbb{R}), \tilde{B}_{2}=\left(\tilde{s}_{i j}\right)_{q \times m}, \tilde{s}_{i j} \in \mathscr{F}^{*}(\mathbb{R})$. 
For the sake of simplicity, we set

$$
\tilde{X} \times \tilde{Y}=\left\{(x, y) ; \tilde{A}_{1} x+\tilde{B}_{1} \precsim \tilde{b}_{1}, \tilde{A}_{2} x+\tilde{B}_{2} y \precsim \tilde{b}_{2}\right\}
$$

and assume that $\tilde{X} \times \tilde{Y}$ is compact. In a FMOLBP problem, for each $(x, y) \in$ $\tilde{X} \times \tilde{Y}$, the value of the objective functions $F(x, y)=\left(F_{1}(x, y), \cdots, F_{s}(x, y)\right)$ and $f(x, y)=\left(f_{1}(x, y), \cdots, f_{t}(x, y)\right)$ of the leader and the follower are $s$ dimensional and $t$-dimensional fuzzy numbers, respectively. Thus, we introduce the following concepts of optimal solutions to the FMOLBP problems.

Definition 11 A point $\left(x^{*}, y^{*}\right) \in \tilde{X} \times \tilde{Y}$ is said to be a complete optimal solution to the FMOLBP problem if it holds that $F\left(x^{*}, y^{*}\right) \precsim F(x, y)$ and $f\left(x^{*}, y^{*}\right) \precsim f(x, y)$ for all $(x, y) \in \tilde{X} \times \tilde{Y}$.

Definition 12 A point $\left(x^{*}, y^{*}\right) \in \tilde{X} \times \tilde{Y}$ is said to be a Pareto optimal solution to the FMOLBP problem if there does not exist $(x, y) \in \tilde{X} \times \tilde{Y}$ such that $F\left(x^{*}, y^{*}\right) \succsim F(x, y)$ and $f\left(x^{*}, y^{*}\right) \succsim f(x, y)$ holds.

Definition 13 A point $\left(x^{*}, y^{*}\right) \in \tilde{X} \times \tilde{Y}$ is said to be a weak Pareto optimal solution to the FMOLBP problem if there is no $(x, y) \in \tilde{X} \times \tilde{Y}$ such that $F\left(x^{*}, y^{*}\right) \succ F(x, y)$ and $f\left(x^{*}, y^{*}\right) \succ f(x, y)$ holds.

Associated with the FMOLBP problem, we now consider the following multiobjective linear bilevel programming (MOLBP) problem:

For $x \in X \subset \mathbb{R}^{n}, y \in Y \subset \mathbb{R}^{m}, F: X \times Y \rightarrow \mathscr{F}^{*}\left(\mathbb{R}^{s}\right)$, and $f: X \times Y \rightarrow$ $\mathscr{F}^{*}\left(\mathbb{R}^{t}\right)$

$$
\begin{gathered}
\min _{x \in X}(F(x, y))_{\lambda}^{L(R)}=\left(\left(F_{1}(x, y)\right)_{\lambda}^{L},\left(F_{1}(x, y)\right)_{\lambda}^{R}, \cdots\left(F_{s}(x, y)\right)_{\lambda}^{L},\left(F_{s}(x, y)\right)_{\lambda}^{R}\right)^{T}, \\
\text { subject to } A_{1 \lambda}{ }_{\lambda}^{L} x+B_{1}{ }_{\lambda}^{L} y \leqq b_{1}{ }_{\lambda}^{L}, A_{1 \lambda}{ }^{R} x+B_{1}{ }_{\lambda}^{R} y \leqq b_{1}{ }_{\lambda}^{R}, \lambda \in[0,1] \\
\min _{y \in Y}(f(x, y))_{\lambda}^{L(R)}=\left(\left(f_{1}(x, y)\right)_{\lambda}^{L},\right. \\
\left.\left(f_{1}(x, y)\right)_{\lambda}^{R}, \cdots,\left(f_{t}(x, y)\right)_{\lambda}^{L},\left(f_{t}(x, y)\right)_{\lambda}^{R}\right)^{T}, \lambda \in[0,1] \\
\text { subject to } A_{2 \lambda}{ }_{\lambda}^{L} x+B_{2}{ }_{\lambda}^{L} y \leqq b_{2 \lambda}{ }_{\lambda}^{L}, A_{2 \lambda}{ }_{\lambda}^{R} x+B_{2 \lambda}{ }_{\lambda}^{R} y \leqq b_{2}{ }_{\lambda}^{R}, \lambda \in[0,1]
\end{gathered}
$$

where $\left(F_{i}(x, y)\right)_{\lambda}^{L}=c_{i 1}^{L} x+d_{i 1}^{L} y,\left(F_{i}(x, y)\right)_{\lambda}^{R}=c_{i 1}{ }_{\lambda}^{R} x+d_{i 1 \lambda}^{R} y,\left(f_{j}(x, y)\right)_{\lambda}^{L}=$ $c_{j 2}{ }_{\lambda}^{L} x+d_{j 12}{ }_{\lambda}^{L} y$ and $\left(f_{j}(x, y)\right)_{\lambda}^{R}=c_{j 2}{ }_{\lambda}^{R} x+d_{j 12}{ }_{\lambda}^{R} y, \lambda \in[0,1], c_{i 1}{ }_{\lambda}^{L}, c_{i 1}{ }_{\lambda}^{R}, c_{j 2}{ }_{\lambda}^{L}, c_{j 2}^{R} \in$ $\mathbb{R}^{n}, d_{i 1}{ }_{\lambda}^{L}, d_{i 1 \lambda}, d_{j 2}{ }_{\lambda}^{L}, d_{j 2}{ }_{\lambda}^{R} \in \mathbb{R}^{m}, d_{i 1}{ }_{\lambda}^{L}, d_{i 1 \lambda}^{R}, d_{j 2_{\lambda}}^{L}, d_{j 2_{\lambda}}^{R} \in \mathbb{R}^{m}, i=1,2, \cdots, s, j=$ $1,2, \cdots, t, b_{1 \lambda}^{L}, b_{1 \lambda}^{R} \in \mathbb{R}^{p}, b_{2 \lambda}^{L}, b_{2 \lambda}^{R} \in \mathbb{R}^{q}, A_{1 \lambda}^{L}=\left(a_{i j_{\lambda}}^{L}\right), A_{1 \lambda}^{R}=\left(a_{i j_{\lambda}}^{R}\right) \in \mathbb{R}^{p \times n}$, $A_{2 \lambda}^{L}=\left(e_{i j_{\lambda}}^{L}\right), A_{2 \lambda}^{R}=\left(e_{i j \lambda}^{R}\right) \in \mathbb{R}^{q \times n}, B_{1 \lambda}^{L}=\left(b_{i j_{\lambda}}^{L}\right), B_{1 \lambda}^{R}=\left(b_{i j_{\lambda}}^{R}\right) \in \mathbb{R}^{p \times m}$, $B_{2 \lambda}^{L}=\left(s_{i j_{\lambda}}^{L}\right), B_{2 \lambda}^{R}=\left(s_{i j_{\lambda}}^{R}\right) \in \mathbb{R}^{q \times m}$. 
For the sake of simplicity, we set

$$
\begin{aligned}
& \underline{X} \times \underline{Y}=\left\{(x, y) ; A_{1 \lambda}^{L} x+B_{1 \lambda}^{L} \leqq b_{1 \lambda}^{L}, A_{1 \lambda}^{R} x+B_{1 \lambda}^{R} \leqq b_{1 \lambda}^{R},\right. \\
& \left.A_{2 \lambda}{ }^{L} x+B_{2 \lambda}{ }_{\lambda}^{L} \leqq b_{2 \lambda}{ }^{L}, A_{2 \lambda}^{R} x+B_{2 \lambda}^{R} \leqq b_{2 \lambda}^{R}\right\}
\end{aligned}
$$

and assume that $\underline{X} \times \underline{Y}$ is compact.

Obviously, $\tilde{X} \times \tilde{Y}=\underline{X} \times \underline{Y}$.

Definition 14 A point $\left(x^{*}, y^{*}\right) \in \underline{X} \times \underline{Y}$ is said to be a complete optimal solution to the MOLBP problem if it holds that

$$
\begin{gathered}
\left(\left(F_{1}\left(x^{*}, y^{*}\right)\right)_{\lambda}^{L},\left(F_{1}\left(x^{*}, y^{*}\right)\right)_{\lambda}^{R}, \cdots,\left(F_{s}\left(x^{*}, y^{*}\right)\right)_{\lambda}^{L},\left(F_{s}\left(x^{*}, y^{*}\right)\right)_{\lambda}^{R}\right)^{T} \\
\leqq\left(\left(F_{1}(x, y)\right)_{\lambda}^{L},\left(F_{1}(x, y)\right)_{\lambda}^{R}, \cdots,\left(F_{s}(x, y)\right)_{\lambda}^{L},\left(F_{s}(x, y)\right)_{\lambda}^{R}\right)^{T}
\end{gathered}
$$

and

$$
\begin{gathered}
\left(\left(f_{1}\left(x^{*}, y^{*}\right)\right)_{\lambda}^{L},\left(f_{1}\left(x^{*}, y^{*}\right)\right)_{\lambda}^{R}, \cdots,\left(f_{t}\left(x^{*}, y^{*}\right)\right)_{\lambda}^{L},\left(f_{t}\left(x^{*}, y^{*}\right)\right)_{\lambda}^{R}\right)^{T} \\
\leqq\left(\left(f_{1}(x, y)\right)_{\lambda}^{L},\left(f_{1}(x, y)\right)_{\lambda}^{R}, \cdots,\left(f_{t}(x, y)\right)_{\lambda}^{L},\left(f_{t}(x, y)\right)_{\lambda}^{R}\right)^{T}
\end{gathered}
$$

for $\lambda \in[0,1]$ and $(x, y) \in \underline{X} \times \underline{Y}$.

Definition 15 A point $\left(x^{*}, y^{*}\right) \in \underline{X} \times \underline{Y}$ is said to be a Pareto optimal solution to the MOLBP problem if there is no $(x, y) \in \underline{X} \times \underline{Y}$ such that

$$
\begin{gathered}
\left(\left(F_{1}\left(x^{*}, y^{*}\right)\right)_{\lambda}^{L},\left(F_{1}\left(x^{*}, y^{*}\right)\right)_{\lambda}^{R}, \cdots,\left(F_{s}\left(x^{*}, y^{*}\right)\right)_{\lambda}^{L},\left(F_{s}\left(x^{*}, y^{*}\right)\right)_{\lambda}^{R}\right)^{T} \\
\geq\left(\left(F_{1}(x, y)\right)_{\lambda}^{L},\left(F_{1}(x, y)\right)_{\lambda}^{R}, \cdots,\left(F_{s}(x, y)\right)_{\lambda}^{L},\left(F_{s}(x, y)\right)_{\lambda}^{R}\right)^{T}
\end{gathered}
$$

or

$$
\begin{gathered}
\left(\left(f_{1}\left(x^{*}, y^{*}\right)\right)_{\lambda}^{L},\left(f_{1}\left(x^{*}, y^{*}\right)\right)_{\lambda}^{R}, \cdots,\left(f_{t}\left(x^{*}, y^{*}\right)\right)_{\lambda}^{L},\left(f_{t}\left(x^{*}, y^{*}\right)\right)_{\lambda}^{R}\right)^{T} \\
\geq\left(\left(f_{1}(x, y)\right)_{\lambda}^{L},\left(f_{1}(x, y)\right)_{\lambda}^{R}, \cdots,\left(f_{t}(x, y)\right)_{\lambda}^{L},\left(f_{t}(x, y)\right)_{\lambda}^{R}\right)^{T}
\end{gathered}
$$

hold.

Definition 16 A point $\left(x^{*}, y^{*}\right) \in \underline{X} \times \underline{Y}$ is said to be a weak Pareto optimal solution to the MOLBP problem if there is no $(x, y) \in \underline{X} \times \underline{Y}$ such that

$$
\begin{gathered}
\left(\left(F_{1}\left(x^{*}, y^{*}\right)\right)_{\lambda}^{L},\left(F_{1}\left(x^{*}, y^{*}\right)\right)_{\lambda}^{R}, \cdots,\left(F_{s}\left(x^{*}, y^{*}\right)\right)_{\lambda}^{L},\left(F_{s}\left(x^{*}, y^{*}\right)\right)_{\lambda}^{R}\right)^{T} \\
>\left(\left(F_{1}(x, y)\right)_{\lambda}^{L},\left(F_{1}(x, y)\right)_{\lambda}^{R}, \cdots,\left(F_{s}(x, y)\right)_{\lambda}^{L},\left(F_{s}(x, y)\right)_{\lambda}^{R}\right)^{T}
\end{gathered}
$$


or

$$
\begin{gathered}
\left(\left(f_{1}\left(x^{*}, y^{*}\right)\right)_{\lambda}^{L},\left(f_{1}\left(x^{*}, y^{*}\right)\right)_{\lambda}^{R}, \cdots,\left(f_{t}\left(x^{*}, y^{*}\right)\right)_{\lambda}^{L},\left(f_{t}\left(x^{*}, y^{*}\right)\right)_{\lambda}^{R}\right)^{T} \\
>\left(\left(f_{1}(x, y)\right)_{\lambda}^{L},\left(f_{1}(x, y)\right)_{\lambda}^{R}, \cdots,\left(f_{t}(x, y)\right)_{\lambda}^{L},\left(f_{t}(x, y)\right)_{\lambda}^{R}\right)^{T}
\end{gathered}
$$

hold.

Theorem 17 Let $\left(x^{*}, y^{*}\right)$ be the optimal solution of the MOLBP problem defined by (2). Then it is also an optimal solution of the FMOLBP problem defined by (1).

Theorem 18 For $x \in X \subset \mathbb{R}^{n}, y \in Y \subset \mathbb{R}^{m}$, if all the fuzzy parameters $\tilde{a}_{i j}$, $\tilde{b}_{i j}, \tilde{e}_{i j}, \tilde{s}_{i j}, \tilde{c}_{i j}, \tilde{b}_{1}, \tilde{b}_{2}$ and $\tilde{d}_{i j}$ have piecewise trapezoidal membership functions in the FMOLBP problem (1),

$$
\mu_{\tilde{z}}(t)= \begin{cases}0, & t<z_{\alpha_{0}}^{L}, \\ \frac{\alpha_{1}-\alpha_{0}}{z_{\alpha_{1}}^{L}-z_{\alpha_{0}}^{L}}\left(t-z_{\alpha_{0}}^{L}\right)+\alpha_{0}, & z_{\alpha_{0}}^{L} \leqslant t<z_{\alpha_{1}}^{L} \\ \frac{\alpha_{1}-\alpha_{0}}{z_{\alpha_{2}}^{L}-z_{\alpha_{1}}^{L}}\left(t-z_{\alpha_{1}}^{L}\right)+\alpha_{1}, & z_{\alpha_{1}}^{L} \leqslant t<z_{\alpha_{2}}^{L} \\ \vdots & \vdots \\ \alpha, & z_{\alpha_{n}}^{L} \leqslant t<z_{\alpha_{n}}^{R} \\ \frac{\alpha_{n}-\alpha_{n-1}}{z_{\alpha_{n-1}}^{R}-z_{\alpha_{n}}^{R}}\left(-t+z_{\alpha_{n-1}}^{R}\right)+\alpha_{n-1}, & z_{\alpha_{n}}^{R} \leqslant t<z_{\alpha_{n-1}}^{R} \\ \vdots & \vdots \\ \frac{\alpha_{0}-\alpha_{1}}{z_{\alpha_{1}}^{R}-z_{\alpha_{0}}^{R}}\left(-t+z_{\alpha_{0}}^{R}\right)+\alpha_{0}, & z_{\alpha_{1}}^{R} \leqslant t \leqslant z_{\alpha_{0}}^{R} \\ 0, & z_{\alpha_{0}}^{R}<t\end{cases}
$$

where $\tilde{z}$ denotes $\tilde{a}_{i j}, \tilde{b}_{i j}, \tilde{e}_{i j}, \tilde{s}_{i j}, \tilde{c}_{i j}, \tilde{b}_{1}, \tilde{b}_{2}$, and $\tilde{d}_{i j}$ respectively, then, $\left(x^{*}, y^{*}\right)$ is a complete optimal solution to the problem (1) if and only if $\left(x^{*}, y^{*}\right)$ is a complete optimal solution to the MOLBP problem:

$$
\begin{gathered}
\min _{x \in X}\left(F_{i}(x, y)\right)_{\alpha_{j}}^{L}=c_{i 1}{ }_{\alpha_{j}}^{L} x+d_{i 1}{ }_{\alpha_{j}}^{L} y, \quad i=1,2, \ldots, s ; j=0,1, \ldots, n \\
\min _{x \in X}\left(F_{i}(x, y)\right)_{\alpha_{j}}^{R}=c_{i 1}{ }_{\alpha_{j}}^{R} x+d_{i 1}{ }_{\alpha_{j}}^{R} y, \quad i=1,2, \ldots, s ; j=0,1, \ldots, n \\
\text { subject to } A_{1}^{L}{ }_{\alpha_{j}}^{L} x+B_{1}{ }_{\alpha_{j}}^{L} y \leqq b_{1}{ }_{\alpha_{j}}^{L}, j=0,1, \ldots, n \\
A_{1}^{R}{ }_{\alpha_{j}}^{R} x+B_{1}^{R}{ }_{\alpha_{j}}^{R} y \leqq b_{1}{ }_{\alpha_{j}}^{R}, j=0,1, \ldots, n \\
\min _{y \in Y}\left(f_{i}(x, y)\right)_{\alpha_{j}}^{L}=c_{i 2}{ }_{\alpha_{j}}^{L} x+d_{i 2}{ }_{\alpha_{j}}^{L} y, i=1,2, \ldots, s ; j=0,1, \ldots, n \\
\min _{y \in Y}\left(f_{i}(x, y)\right)_{\alpha_{j}}^{R}=c_{i 2}{ }_{\alpha_{j}}^{R} x+d_{i 2}{ }_{\alpha_{j}}^{R} y, i=1,2, \ldots, s ; j=0,1, \ldots, n
\end{gathered}
$$




$$
\begin{array}{r}
\text { subject to } A_{2 \alpha_{j}}{ }^{L} x+B_{2}{ }_{\alpha_{j}}^{L} y \leqq b_{2}{ }_{\alpha_{j}}, j=0,1, \ldots, n \\
A_{2 \alpha_{j}}^{R} x+B_{2 \alpha_{j}}{ }^{R} y \leqq b_{2}{ }_{2}^{R}, j=0,1, \ldots, n .
\end{array}
$$

We note

$$
\begin{aligned}
\bar{A}_{1} x+\bar{B}_{1} y & \leqq \bar{b}_{1} \\
\bar{A}_{2} x+\bar{B}_{2} y & \leqq \bar{b}_{2}
\end{aligned}
$$

where

$$
\begin{aligned}
& \bar{A}_{1}=\left(A_{1_{\alpha_{0}}}^{L}, \cdots, A_{1_{\alpha_{n}}}^{L}, A_{1_{\alpha_{0}}}^{R}, \cdots, A_{1_{\alpha_{n}}}^{R}\right)^{T}, \\
& \bar{A}_{2}=\left(A_{2 \alpha_{0}}^{L}, \cdots, A_{2}^{L} \stackrel{L}{L}, A_{\alpha_{n}}^{R}, \cdots, A_{2 \alpha_{0}}^{R}, \cdots\right)^{T} \text {, } \\
& \bar{B}_{1}=\left(B_{1}{ }_{\alpha_{0}}^{L}, \cdots, B_{1} \alpha_{\alpha_{n}}^{L}, B_{1 \alpha_{0}}^{R}, \cdots, B_{1 \alpha_{n}}^{R}\right)^{T} \text {, } \\
& \bar{B}_{2}=\left(B_{2 \alpha_{0}}^{L}, \cdots, B_{2}^{L} \underset{\alpha_{n}}{L}, B_{2}{ }_{\alpha_{0}}^{R}, \cdots, B_{2}{ }_{\alpha_{n}}^{R}\right)^{T} \text {, } \\
& \bar{b}_{1}=\left(b_{1_{\alpha_{0}}}^{L}, \cdots, b_{1_{\alpha_{n}}}^{L}, b_{1_{\alpha_{0}}}^{R}, \cdots, b_{1_{\alpha_{n}}}^{R}\right)^{T} \text {, } \\
& \bar{b}_{2}=\left(b_{2}{ }_{\alpha_{0}}^{L}, \cdots, b_{2}{ }_{\alpha_{n}}^{L}, b_{2}{ }_{\alpha_{0}}^{R}, \cdots, b_{2}{ }_{\alpha_{n}}^{R}\right)^{T} \text {. }
\end{aligned}
$$

Then we can re-write (4) by using

$$
\begin{aligned}
& \min _{x \in X}\left(F_{i}(x, y)\right)_{\alpha_{j}}^{L}=c_{i 1_{\alpha_{j}}}^{L} x+d_{i 1_{\alpha_{j}}}^{L} y, i=1,2, \ldots, s ; j=0,1, \ldots, n \\
& \min _{x \in X}\left(F_{i}(x, y)\right)_{\alpha_{j}}^{R}=c_{i 1}{ }_{\alpha_{j}}^{R} x+d_{i 1}{ }_{\alpha_{j}}^{R} y, i=1,2, \ldots, s ; j=0,1, \ldots, n \\
& \min _{y \in Y}\left(f_{i}(x, y)\right)_{\alpha_{j}}^{L}=c_{i 1}{ }_{\alpha_{j}}^{L} x+d_{i 1_{\alpha_{j}}}^{L} y, i=1,2, \ldots, s ; j=0,1, \ldots, n \\
& \min _{y \in Y}\left(f_{i}(x, y)\right)_{\alpha_{j}}^{R}=c_{i 1}^{R}{ }_{\alpha_{j}}^{R} x+d_{i 1_{\alpha_{j}}}^{R} y, i=1,2, \ldots, s ; j=0,1, \ldots, n \\
& \text { subject to } \bar{A}_{2} x+\bar{B}_{2} y \leqq \bar{b}_{2} \text {. }
\end{aligned}
$$

Theorem 19 For $x \in X \subset \mathbb{R}^{n}, y \in Y \subset \mathbb{R}^{m}$, if all the fuzzy parameters $\tilde{a}_{i j}$, $\tilde{b}_{i j}, \tilde{e}_{i j}, \tilde{s}_{i j}, \tilde{c}_{i j}$, and $\tilde{d}_{i j}$ have piecewise trapezoidal membership functions (3) in the FMOLBP problem (1), then $\left(x^{*}, y^{*}\right)$ is a Pareto optimal solution to the problem (1) if and only if $\left(x^{*}, y^{*}\right)$ is a Pareto optimal solution to the MOLBP problem $\left(4^{\prime}\right)$.

PROOF. Let $\left(x^{*}, y^{*}\right)$ be a Pareto optimal solution to the FMOLBLP problem. On the contrary, we suppose that there exists a $(\bar{x}, \bar{y}) \in X \times Y$ such that, for $\lambda=\alpha, \beta$

$$
\begin{gathered}
\left(\left(F_{1}\left(x^{*}, y^{*}\right)\right)_{\lambda}^{L},\left(F_{1}\left(x^{*}, y^{*}\right)\right)_{\lambda}^{R}, \cdots,\left(F_{s}\left(x^{*}, y^{*}\right)\right)_{\lambda}^{L},\left(F_{s}\left(x^{*}, y^{*}\right)\right)_{\lambda}^{R}\right)^{T} \\
\geq\left(\left(F_{1}(\bar{x}, \bar{y})\right)_{\lambda}^{L},\left(F_{1}(\bar{x}, \bar{y})\right)_{\lambda}^{R},\left(F_{s}(\bar{x}, \bar{y})\right)_{\lambda}^{L},\left(F_{s}(\bar{x}, \bar{y})\right)_{\lambda}^{R}\right)^{T} .
\end{gathered}
$$


Therefore

$$
\begin{gathered}
0 \geqslant\left(\left(F_{1}(\bar{x}, \bar{y})\right)_{\lambda}^{L}-\left(F_{1}\left(x^{*}, y^{*}\right)\right)_{\lambda}^{L},\left(F_{1}(\bar{x}, \bar{y})\right)_{\lambda}^{R}\left(F_{1}\left(x^{*}, y^{*}\right)\right)_{\lambda}^{R}, \cdots\right. \\
\left.\left(F_{s}(\bar{x}, \bar{y})\right)_{\lambda}^{L}-\left(F_{s}\left(x^{*}, y^{*}\right)\right)_{\lambda}^{L},\left(F_{s}(\bar{x}, \bar{y})\right)_{\lambda}^{R}-\left(F_{s}\left(x^{*}, y^{*}\right)\right)_{\lambda}^{R}\right)^{T} .
\end{gathered}
$$

Hence

$0 \geqslant\left(F_{i}(\bar{x}, \bar{y})\right)_{\lambda}^{L}-\left(F_{i}\left(x^{*}, y^{*}\right)\right)_{\lambda}^{L}, 0 \geqslant\left(F_{i}(\bar{x}, \bar{y})\right)_{\lambda}^{R}-\left(F_{i}\left(x^{*}, y^{*}\right)\right)_{\lambda}^{R}, i=1,2, \cdots, s$.

That is

$$
\left(F_{i}(\bar{x}, \bar{y})\right)_{\lambda}^{L} \leq\left(F_{i}\left(x^{*}, y^{*}\right)\right)_{\lambda}^{L},\left(F_{i}(\bar{x}, \bar{y})\right)_{\lambda}^{R} \leq\left(F_{i}\left(x^{*}, y^{*}\right)\right)_{\lambda}^{R}, i=1,2, \cdots, s .
$$

Consequently, for any $\lambda \in[\beta, \alpha]$, we have

$$
\left(F_{i}(\bar{x}, \bar{y})\right)_{\lambda}^{L} \leq\left(F_{i}\left(x^{*}, y^{*}\right)\right)_{\lambda}^{L},\left(F_{i}(\bar{x}, \bar{y})\right)_{\lambda}^{R} \leq\left(F_{i}\left(x^{*}, y^{*}\right)\right)_{\lambda}^{R}, i=1,2, \cdots, s .
$$

that is $F\left(x^{*}, y^{*}\right) \succeq F(\bar{x}, \bar{y})$. However, this contradicts the assumption that $\left(x^{*}, y^{*}\right)$ is a Pareto optimal solution to the FMOLBLP problem.

Let $\left(x^{*}, y^{*}\right)$ be a Pareto optimal solution to the MOLBLP problem. If $\left(x^{*}, y^{*}\right)$ is not a Pareto optimal solution to the problem, then there exists $(\bar{x}, \bar{y}) \in X \times Y$ such that $F\left(x^{*}, y^{*}\right) \succeq F(\bar{x}, \bar{y})$. Therefore, for any $\lambda \in[\beta, \alpha]$, we have

$$
\begin{gathered}
\left(\left(F_{1}\left(x^{*}, y^{*}\right)\right)_{\lambda}^{L},\left(F_{1}\left(x^{*}, y^{*}\right)\right)_{\lambda}^{R}, \cdots,\left(F_{s}\left(x^{*}, y^{*}\right)\right)_{\lambda}^{L},\left(F_{s}\left(x^{*}, y^{*}\right)\right)_{\lambda}^{R}\right)^{T} \\
\geq\left(\left(F_{1}(\bar{x}, \bar{y})\right)_{\lambda}^{L},\left(F_{1}(\bar{x}, \bar{y})\right)_{\lambda}^{R},\left(F_{s}(\bar{x}, \bar{y})\right)_{\lambda}^{L},\left(F_{s}(\bar{x}, \bar{y})\right)_{\lambda}^{R}\right)^{T} .
\end{gathered}
$$

that is

$$
\left(F_{i}(\bar{x}, \bar{y})\right)_{\lambda}^{L} \leq\left(F_{i}\left(x^{*}, y^{*}\right)\right)_{\lambda}^{L},\left(F_{i}(\bar{x}, \bar{y})\right)_{\lambda}^{R} \leq\left(F_{i}\left(x^{*}, y^{*}\right)\right)_{\lambda}^{R}, i=1,2, \cdots, s .
$$

Hence, for $\lambda=\alpha$ and $\lambda=\beta$, we have

$$
\left(F_{i}(\bar{x}, \bar{y})\right)_{\lambda}^{L} \leq\left(F_{i}\left(x^{*}, y^{*}\right)\right)_{\lambda}^{L},\left(F_{i}(\bar{x}, \bar{y})\right)_{\lambda}^{R} \leq\left(F_{i}\left(x^{*}, y^{*}\right)\right)_{\lambda}^{R}, i=1,2, \cdots, s .
$$

which contradicts the assumption that $\left(x^{*}, y^{*}\right)$ is a Pareto optimal solution to the MOLBLP problem.

Theorem 20 For $x \in X \subset \mathbb{R}^{n}, y \in Y \subset \mathbb{R}^{m}$, if all the fuzzy parameters $\tilde{a}_{i j}$, $\tilde{b}_{i j}, \tilde{e}_{i j}, \tilde{s}_{i j}, \tilde{c}_{i j}$, and $\tilde{d}_{i j}$ have piecewise trapezoidal membership functions (3) in the FMOLBP problem (1), then $\left(x^{*}, y^{*}\right)$ is a weak Pareto optimal solution to the problem (1) if and only if $\left(x^{*}, y^{*}\right)$ is a weak Pareto optimal solution to the MOLBP problem (4').

PROOF. See Theorem 19. 
Theorem 21 For $x \in X \subset \mathbb{R}^{n}, y \in Y \subset \mathbb{R}^{m}$, if all the fuzzy parameters $\tilde{a}_{i j}$, $\tilde{b}_{i j}, \tilde{e}_{i j}, \tilde{s}_{i j}, \tilde{c}_{i j}$, and $\tilde{d}_{i j}$ have piecewise trapezoidal membership functions (3) in the FMOLBP problem (1), then a necessary and sufficient condition that $\left(x^{*}, y^{*}\right)$ solves the FMOLBP problem (1) is that there exist (row) vectors $u^{*}$, $v^{*}$ and $z^{*}$ such that $\left(x^{*}, y^{*}, u^{*}, v^{*}, z^{*}\right)$ solves:

$$
\begin{aligned}
& \min _{x \in X}=\sum_{j=1}^{s} w_{j 1}\left(\sum_{i=0}^{n}\left(c_{j 1}^{L}{ }_{\alpha_{i}}^{L} x+d_{j 1}{ }_{\alpha_{i}}^{L} y\right)+\sum_{i=0}^{n}\left(c_{j 1}^{R} x+d_{j \alpha_{\alpha_{i}}}^{R} y\right)\right) \\
& \text { subject to } \bar{A}_{1} x+\bar{B}_{1} y \leqq \bar{b}_{1} \\
& \bar{A}_{2} x+\bar{B}_{2} y \leqq \bar{b}_{2} \\
& u\left(\sum_{i=0}^{n} B_{1 \alpha_{i}}^{L}+\sum_{i=0}^{n} B_{1 \alpha_{i}}^{R}\right)+v\left(\sum_{i=0}^{n} B_{2}^{L}{ }_{\alpha_{i}}^{L}+\sum_{i=0}^{n} B_{2 \alpha_{i}}^{R}\right)-z \\
& =-\sum_{j=1}^{t} w_{j 2}\left(\sum_{i=0}^{n} d_{j 2}{ }_{\alpha_{i}}^{L}+\sum_{i=0}^{n} d_{j 2} R\right. \\
& u\left(\left(\sum_{i=0}^{n} b_{1 \alpha_{i}}^{L}+\sum_{i=0}^{n} b_{1 \alpha_{i}}^{R}\right)-\left(\sum_{i=0}^{n} A_{1 \alpha_{i}}^{L}+\sum_{i=0}^{n} A_{1 \alpha_{i}}^{R}\right) x\right. \\
& \left.-\left(\sum_{i=0}^{n} B_{1}{ }_{\alpha_{i}}^{L}+\sum_{i=0}^{n} B_{1}{ }_{1 \alpha_{i}}^{R}\right) y\right)+v\left(\left(\sum_{i=0}^{n} b_{2}{ }_{2}^{L}+\sum_{i=0}^{n} b_{2}{ }_{2}^{R}\right)\right. \\
& \left.-\left(\sum_{i=0}^{n} A_{2 \alpha_{i}}^{L}+\sum_{i=0}^{n} A_{2 \alpha_{i}}^{R}\right) x-\left(\sum_{i=0}^{n} B_{2}{ }_{2}^{L}+\sum_{i=0}^{n} B_{2}{ }_{2}^{R}\right) y\right) \\
& +z y=0 \\
& x \geq 0, y \geq 0, u \geq 0, v \geq 0, z \geq 0, \sum_{j=1}^{s} w_{j 1}=1 \text { and } \sum_{j=1}^{t} w_{j 2}=1 .
\end{aligned}
$$

PROOF. We can prove this result by combining Theorem 18 and Theorem 2 of reference [22], and use the weighting method [13].

Obviously, Theorem 21 provides a way to solve FMOLBP problem. Based on this theorem, we can present an approximation branch-and-bound algorithm for solving the FMOLBP problem shown in (1).

\section{An Approximation Branch-and-Bound Algorithm}

This section proposes an approximation branch-and-bound algorithm for solving the FMOLBP problems. 
We first write all the inequalities (except of the leader's variables) of (4'a)$\left(4^{\prime} \mathrm{d}\right)$ as $g_{i}(x, y) \geq 0, i=1, \ldots, p+q+m$, and note that complementary slackness simply means $u_{i} g_{i}(x, y)=0(i=1, \ldots, p+q+m)$. We suppress the complementary term and solve the resulted linear sub-problem. At each time of iteration the condition (5e) is checked. If it is satisfied, the corresponding point is in the inducible region and hence a potential solution to $\left(4^{\prime}\right)$. Otherwise, a branch-and-bound scheme is used to implicitly examine all combinations of the complementarities slackness.

Now, we give some notations for describing the details of the approximation branch-and-bound algorithm.

Let $W=\{1, \ldots, p+q+m\}$ be the index set for the terms in (5e), $\bar{F}$ be the incumbent upper bound on the objective function of the leader. At the $k$ th level of an search tree we define a subset of indices $W_{k} \subset W$, and a path $P_{k}$ corresponding to an assignment of either $u_{i}=0$ or $g_{i}=0$ for $i \in W_{k}$. Now let

$$
\begin{aligned}
S_{k}^{+} & =\left\{i: i \in W_{k}, u_{i}=0\right\} \\
S_{k}^{-} & =\left\{i: i \in W_{k}, g_{i}=0\right\} \\
S_{k}^{0} & =\left\{i: i \notin W_{k}\right\} .
\end{aligned}
$$

For $i \in S_{k}^{0}$, the variables $u_{i}$ or $g_{i}$ are free to assume any nonnegative value in the solution of (5) with (5e) omitted, so complementary slackness will not necessarily be satisfied.

By using these notations we give all steps of the approximation branch-andbound algorithm in Table 1.

We give some explanations for these steps and their working process as follows.

After initialization, Step 7 is designed to find a new point which is potentially bilevel feasible. If no solution exists, or the solution does not offer an improvement over the incumbent (Step 8), the algorithm goes to Step 11 and backtracks.

Step 9 checks the value of $u_{i}^{k} g_{i}\left(x^{k}, y^{k}\right)$ to determine if the complementary slackness conditions are satisfied. In practice, if $\left|u_{i}^{k} g_{i}\right|<10^{-6}$ it is considered to be zero. Confirmation indicates that a feasible solution of a bilevel program has been found and at Step 10 the upper bound on the leader's objective function is updated. Alternatively, if the complementary slackness conditions are not satisfied, the term with the largest product is used at Step 9 to provide a branching variable. Branching is always completed on the Kuhn-Tucker multiplier [2].

At Step 11, the backtracking operation is performed. Note that a live node is one associated with a sub-problem that has not yet been fathomed at either 
Table 1

An approximation branch-and-bound algorithm for FMOLBP problems

\begin{tabular}{|c|c|}
\hline Step 1 & $\begin{array}{l}\text { Given two sets of weights } w_{j 1}(j=1,2, \ldots, s) \text { and } w_{j 2}(j=1,2, \ldots, t) \\
\text { to the objectives of the leader and the follower respectively, and let } \\
\sum_{j=1}^{s} w_{j 1}=1 \text { and } \sum_{j=1}^{t} w_{j 2}=1 .\end{array}$ \\
\hline Step 2 & The problem (1) is transformed to the problem $\left(4^{\prime}\right)$ \\
\hline Step 3 & $\begin{array}{l}\text { Set } l=1 \text {, a range of errors } \epsilon>0 \text {, to solve }(\mathrm{MOLBP})_{2}^{l} \text {, i.e. }\left(4^{\prime}\right) \text { by } \\
\text { using extended branch-and-bound algorithm }[23] \text {. }\end{array}$ \\
\hline Step 4 & $\begin{array}{l}\text { Let the interval }[0,1] \text { be decomposed into } 2^{l-1} \text { equal sub-intervals with } \\
\left(2^{l-1}+1\right) \text { nodes } \lambda_{i}\left(i=0, \cdots, 2^{l-1}\right) \text { which are arranged in the order } \\
\text { of } 0=\lambda_{0}<\lambda_{1}<\cdots<\lambda_{2^{l-1}}=1\end{array}$ \\
\hline Step 5 & $\begin{array}{l}\text { Transform the problem }\left(4^{\prime}\right) \text { to the linear BP problem }(5) \text { by using } \\
\text { Theorem } 17 \text { and weighting method }[13]\end{array}$ \\
\hline Step 6 & $\begin{array}{l}\text { (Initialization) Set } k=0, S_{k}^{+}=\phi, S_{k}^{-}=\phi, S_{k}^{0}=\{1, \ldots, p+q+m\} \text {, } \\
\text { and } \bar{F}=\infty \text {. }\end{array}$ \\
\hline Step 7 & $\begin{array}{l}\text { (Iteration } k \text { ) Set } u_{i}=0 \text { for } i \in S_{k}^{+} \text {and } g_{i}=0 \text { for } i \in S_{k}^{-} \text {. It first } \\
\text { attempts to solve }(5) \text { without }(5 \mathrm{e}) \text {. If the resultant problem is infea- } \\
\text { sible, go to Step } 11 \text {; otherwise, put } k \leftarrow k+1 \text { and label the solution } \\
\left(x^{k}, y^{k}, u^{k}\right) \text {. }\end{array}$ \\
\hline Step 8 & (Fathoming) If $F\left(x^{k}, y^{k}\right) \geqslant \bar{F}$, then go to Step 11 . \\
\hline Step 9 & $\begin{array}{l}\text { (Branching) If } u_{i}^{k} g_{i}\left(x^{k}, y^{k}\right)=0, i=1, \ldots, p+q+m \text {, then go to Step } \\
\text { 10. Otherwise select } i \text { for which } u_{i}^{k} g_{i}\left(x^{k}, y^{k}\right) \neq 0 \text { is the largest and } \\
\text { label it } i_{1} \text {. Put } S_{k}^{+} \leftarrow S_{k}^{+} \cup\left\{i_{1}\right\}, S_{k}^{0} \leftarrow S_{k}^{0} \backslash\left\{i_{1}\right\}, S_{k}^{-} \leftarrow S_{k}^{-} \text {, append } \\
i_{1} \text { to } P_{k} \text {, and go to } \operatorname{Step} 7 \text {. }\end{array}$ \\
\hline Step 10 & (Updating) Let $\bar{F} \leftarrow F\left(x^{k}, y^{k}\right)$. \\
\hline Step 11 & $\begin{array}{l}\text { (Backtracking) If no live node exists, go to Step } 12 \text {. Otherwise branch } \\
\text { to the newest live vertex and update } S_{k}^{+}, S_{k}^{-}, S_{k}^{0} \text { and } P_{k} \text { as discussed } \\
\text { below. Go back to Step } 7 \text {. }\end{array}$ \\
\hline Step 12 & $\begin{array}{l}\text { (Termination) If } \bar{F}=\infty \text {, there is not feasible solution to }(\mathrm{MOLBP})_{2}^{l} \text {. } \\
\text { Otherwise, declare the feasible point associated with } \bar{F} \text { which is the } \\
\text { optimal solution }(x, y)_{2^{l}} \text { to }(\mathrm{MOLBP})_{2}^{l} \text {. }\end{array}$ \\
\hline Step 13 & $l=l+1$, repeat Step 4 to Step 12 . \\
\hline Step 14 & $\begin{array}{l}\text { If }\left\|(x, y)_{2^{l+1}}-(x, y)_{2^{l}}\right\|<\varepsilon \text {, then the solution }\left(x^{*}, y^{*}\right) \text { of the } \\
\text { FMOLBP problem is }(x, y)_{2^{l+1}} \text { Otherwise, go back to Step } 13 \text {. }\end{array}$ \\
\hline Step 15 & Show the result of problem (1). Terminates. \\
\hline
\end{tabular}

Step 7 due to infeasibility or at Step 8 due to bounding, and whose solution violates at least one complementary slackness condition. To facilitate book keeping, the path $P_{k}$ in the branch-and-bound tree is represented by a vector, its dimension is the current depth of the tree. The order of the components of 
$P_{k}$ is determined by their level in the tree. Indices only appear in $P_{k}$ if they are in either $S_{k}^{+}$or $S_{k}^{-}$with the entries underlined if they are in $S_{k}^{-}$. Because the algorithm always branches on a Kuhn-Tucker multiplier first, backtracking is accomplished by finding the rightmost non-underlined component if $P_{k}$, underlining it, and erasing all entries to the right. The erased entries are deleted from $S_{k}^{-}$and added to $S_{k}^{0}$.

\section{Case Based Examples}

We first apply the proposed approximation branch-and-bound algorithm to solve a simple FMOLBP problem to illustrate how the algorithm is used.

Example 22 Consider the following FMOLBP problem with $x \in \mathbb{R}^{1}, y \in \mathbb{R}^{1}$, and $X=\{x \geq 0\}, Y=\{y \geq 0\}$,

$$
\begin{aligned}
& \min _{x \in X} F_{1}(x, y)=-\tilde{1} x+\tilde{2} y \\
& \min _{x \in X} F_{2}(x, y)=\tilde{2} x-\tilde{4} y \\
& \text { subject to }-\tilde{1} x+\tilde{3} y \leq \tilde{4} \\
& \min _{y \in Y} f_{1}(x, y)=-\tilde{1} x+\tilde{2} y \\
& \min _{y \in Y} f_{2}(x, y)=\tilde{2} x-\tilde{1} y \\
& \text { subject to } \tilde{1} x-\tilde{1} y \leq \tilde{0} \\
& \quad-\tilde{1} x-\tilde{1} y \leq \tilde{0}
\end{aligned}
$$

where

$$
\begin{aligned}
& \mu_{\tilde{1}}(t)=\left\{\begin{array}{ll}
0, & t<0, \\
t^{2}, & 0 \leqq t<1, \\
2-t, & 1 \leqq t<2, \\
0, & 2 \leqq t .
\end{array} \quad \mu_{\tilde{2}}(t)= \begin{cases}0, & t<1, \\
t-1, & 1 \leqq t<2, \\
3-t, & 2 \leqq t<3, \\
0, & 3 \leqq t\end{cases} \right. \\
& \mu_{\tilde{3}}(t)=\left\{\begin{array}{ll}
0, & t<2, \\
t-2, & 2 \leqq t<3, \\
4-t, & 3 \leqq t<4, \\
0, & 4 \leqq t .
\end{array} \quad \mu_{\tilde{4}}(t)= \begin{cases}0, & t<3, \\
t-3, & 3 \leqq t<4, \\
5-t, & 4 \leqq t<5, \\
0, & 5 \leqq t .\end{cases} \right.
\end{aligned}
$$




$$
\mu_{\tilde{0}}(t)= \begin{cases}0, & t<-1 \\ t+1, & -1 \leqq t<0 \\ 1-t^{2}, & 0 \leqq t<1 \\ 0, & 1 \leqq t\end{cases}
$$

We now solve this problem by using the proposed approximation branch-andbound algorithm.

Step 1 given the weights $(0.5,0.5)$ for the two fuzzy objectives of the leader and of the follower respectively.

Step 2 The FMOLBP problem is transformed to the following MOLBP problem by using Theorem 17.

$$
\begin{aligned}
& \min _{x \in X}\left(F_{1}(x, y)\right)_{\lambda}^{L}=(-\tilde{1})_{\lambda}^{L} x+\tilde{2}_{\lambda}^{L} y, \quad \lambda \in[0,1] \\
& \min _{x \in X}\left(F_{1}(x, y)\right)_{\lambda}^{R}=(-\tilde{1})_{\lambda}^{R} x+\tilde{2}_{\lambda}^{R} y, \quad \lambda \in[0,1] \\
& \min _{x \in X}\left(F_{2}(x, y)\right)_{\lambda}^{L}=\tilde{2}_{\lambda}^{L} x+(-\tilde{4})_{\lambda}^{L} y, \quad \lambda \in[0,1] \\
& \min _{x \in X}\left(F_{2}(x, y)\right)_{\lambda}^{R}=\tilde{2}_{\lambda}^{R} x+(-\tilde{4})_{\lambda}^{R} y, \quad \lambda \in[0,1] \\
& \text { subject to }(-\tilde{1})_{\lambda}^{L} x+\tilde{3}_{\lambda}^{L} y \leqq \tilde{4}_{\lambda}^{L},(-\tilde{1})_{\lambda}^{R} x+\tilde{3}_{\lambda}^{R} y \leqq \tilde{4}_{\lambda}^{R}, \lambda \in[0,1] \\
& \min _{y \in Y}\left(f_{1}(x, y)\right)_{\lambda}^{L}=\tilde{2}_{\lambda}^{L} x+(-\tilde{1})_{\lambda}^{L} y, \quad \lambda \in[0,1] \\
& \min _{y \in Y}\left(f_{1}(x, y)\right)_{\lambda}^{R}=\tilde{2}_{\lambda}^{R} x+(-\tilde{1})_{\lambda}^{R} y, \quad \lambda \in[0,1] \\
& \min _{y \in Y}\left(f_{2}(x, y)\right)_{\lambda}^{L}=(-\tilde{1})_{\lambda}^{L} x+\tilde{2}_{\lambda}^{L} y, \quad \lambda \in[0,1] \\
& \min _{y \in Y}\left(f_{2}(x, y)\right)_{\lambda}^{R}=(-\tilde{1})_{\lambda}^{R} x+\tilde{2}_{\lambda}^{R} y, \quad \lambda \in[0,1] \\
& \operatorname{subject~to~} \tilde{1}_{\lambda}^{L} x+(-\tilde{1})_{\lambda}^{L} y \leqq \tilde{0}_{\lambda}^{L}, \tilde{1}_{\lambda}^{R} x+(-\tilde{1})_{\lambda}^{R} y \leqq \tilde{0}_{\lambda}^{R}, \quad \lambda \in[0,1] \\
& \quad(-\tilde{1})_{\lambda}^{L} x+(-\tilde{1})_{\lambda}^{L} y \leqq \tilde{0}_{\lambda}^{L}, \quad(-\tilde{1})_{\lambda}^{R} x+(-\tilde{1})_{\lambda}^{R} y \leqq \tilde{0}_{\lambda}^{R}, \quad \lambda \in[0,1]
\end{aligned}
$$

Step 3. Let $l=1$ and a range of errors $\varepsilon=10^{-6}>0$.

Step 4. Let the interval $[0,1]$ be decomposed into $2^{l-1}$ equal sub-intervals with $\left(2^{l-1}+1\right)$ nodes $\lambda_{i},\left(i=0, \cdots, 2^{l-1}\right)$ which is arranged in the order of $0=\lambda_{0}<\lambda_{1}<\cdots<\lambda_{2^{l-1}}=1$. We solve the following MOLBP problem

$$
\begin{aligned}
& \min _{x \in X}\left(F_{1}(x, y)\right)_{1}^{L(R)}=-1 x+2 y \\
& \min _{x \in X}\left(F_{1}(x, y)\right)_{0}^{L}=-2 x+y \\
& \min _{x \in X}\left(F_{1}(x, y)\right)_{0}^{R}=0 x+3 y
\end{aligned}
$$




$$
\begin{aligned}
& \min _{x \in X}\left(F_{2}(x, y)\right)_{1}^{L(R)}=2 x-4 y \\
& \min _{x \in X}\left(F_{2}(x, y)\right)_{0}^{L}=1 x-5 y \\
& \min _{x \in X}\left(F_{2}(x, y)\right)_{0}^{R}=3 x-3 y \\
& \text { subject to }-1 x+3 y \leq 4 \\
& -2 x+2 y \leq 3 \\
& 0 x+4 y \leq 5 \\
& \min _{y \in Y}\left(f_{1}(x, y)\right)_{1}^{L(R)}=2 x-1 y \\
& \min _{y \in Y}\left(f_{1}(x, y)\right)_{0}^{L}=1 x-2 y \\
& \min _{y \in Y}\left(f_{1}(x, y)\right)_{0}^{R}=3 x-0 y \\
& \min _{y \in Y}\left(f_{2}(x, y)\right)_{1}^{L(R)}=-1 x+2 y \\
& \min _{y \in Y}\left(f_{2}(x, y)\right)_{0}^{L}=-2 x+1 y \\
& \min _{y \in Y}\left(f_{2}(x, y)\right)_{0}^{L}=0 x+3 y \\
& \operatorname{subject}_{1} \text { to } 1 x-1 y \leq 0 \\
& 0 x-2 y \leq-1 \\
& 2 x-0 y \leq 1 \\
& -1 x-1 y \leq 0 \\
& -2 x-2 y \leq-1 .
\end{aligned}
$$

Step 5. We transform this MOLBP problem to the linear BP problem by using the method of weighting.

$$
\begin{aligned}
& \min _{x \in X} F(x, y)=3 x-6 y \\
& \text { subject to }-1 x+3 y \leq 4 \\
& -2 x+2 y \leq 3 \\
& 0 x+4 y \leq 5 \\
& \min _{y \in Y} f(x, y)=3 x+3 y \\
& \text { subject to } 1 x-1 y \leq 0 \\
& 0 x-2 y \leq-1 \\
& 2 x-0 y \leq 1 \\
& -1 x-1 y \leq 0 \\
& -2 x-2 y \leq-1 .
\end{aligned}
$$

Step 6-12. According to the proposed extended branch-and-bound algorithm, let us rewrite it as follows in (3), we have 


$$
\begin{aligned}
& g_{1}(x, y)=4-(-1 x+3 y) \geq 0 \\
& g_{2}(x, y)=3-(-2 x+2 y) \geq 0 \\
& g_{3}(x, y)=5-(0 x+4 y) \geq 0 \\
& g_{4}(x, y)=-(1 x-1 y) \geq 0 \\
& g_{5}(x, y)=-1-(0 x-2 y) \geq 0 \\
& g_{6}(x, y)=1-(2 x-0 y) \geq 0 \\
& g_{7}(x, y)=1 x+1 y \geq 0 \\
& g_{8}(x, y)=-1-(-2 x-2 y) \geq 0 \\
& g_{9}(x, y)=y \geq 0
\end{aligned}
$$

and also have

$$
\begin{aligned}
\min _{x \in X} F(x, y) & =3 x-6 y \\
\text { subject to } & -1 x+3 y \leq 4 \\
& -2 x+2 y \leq 3 \\
& 0 x+4 y \leq 5 \\
& 1 x-1 y \leq 0 \\
& 0 x-2 y \leq-1 \\
& 2 x-0 y \leq 1 \\
& -1 x-1 y \leq 0 \\
& -2 x-2 y \leq-1 \\
& 3 u_{1}+2 u_{2}+4 u_{3}-u_{4}-2 u_{5}-0 u_{6}-u_{7}-2 u_{8}-u_{9}=-3 \\
& \sum^{9} u_{i} g_{i}(x, y)=0 \\
& x \geqslant 0, y \geqslant 0, u_{1} \geqslant 0, \ldots, u_{9} \geqslant 0 .
\end{aligned}
$$

Finally, we get the following linear programming problem with one check condition.

$$
\begin{gathered}
\min _{x \in X} F(x, y)=3 x-6 y \\
\text { subject to }-1 x+3 y \leq 4 \\
-2 x+2 y \leq 3 \\
0 x+4 y \leq 5 \\
1 x-1 y \leq 0 \\
\\
0 x-2 y \leq-1 \\
\\
2 x-0 y \leq 1 \\
-1 x-1 y \leq 0
\end{gathered}
$$




$$
\begin{aligned}
& -2 x-2 y \leq-1 \\
& 3 u_{1}+2 u_{2}+4 u_{3}-u_{4}-2 u_{5}-0 u_{6}-u_{7}-2 u_{8}-u_{9}=-3 \\
& x \geqslant 0, y \geqslant 0, u_{1} \geqslant 0, \ldots, u_{9} \geqslant 0 .
\end{aligned}
$$

At each time of iteration, the following condition is checked.

$$
\sum_{i=1}^{9} u_{i} g_{i}(x, y)=0 \text {. }
$$

More specifically, after initializing the data, the algorithm finds a feasible solution to the Kuhn-Tucker representation with the complementary slackness conditions omitted and proceeds to Step 9. The current point, $x^{1}=0$, $y^{1}=1.25, u^{1}=(0,0,0,3,0,0,0,0,0)$, with $F\left(x^{1}, y^{1}\right)=-7.5$ is not satisfied complementarities so a branching variable is selected $\left(u_{4}\right)$ and the index sets are updated, giving $S_{1}^{+}=\{4\}, S_{1}^{-}=\phi, S_{1}^{0}=\{1,2,3,5,6,7,8,9\}$ and $P_{1}=\{4\}$.

In the next four iterations, the algorithm branches on $u_{5}, u_{7}, u_{8}$ and $u_{9}$, respectively. Now, five levels down in the branch-and-bound search tree (Fig. 1), the current sub-problem at Step 7 turns out to be infeasible so the algorithm goes to Step 11 and backtracks. The index sets are $S_{5}^{+}=\{4,5,7,8\}, S_{5}^{-}=\{9\}$, $S_{5}^{0}=\{1,2,3,6\}$ and $P_{5}=\{4,5,7,8, \underline{9}\}$.

So we go to Step 7, and the algorithm turns out to be infeasible, so the algorithm goes to Step 11 and backtracks. The index sets are $S_{6}^{+}=\{4,5,7\}$, $S_{6}^{-}=\{8\}, S_{6}^{0}=\{1,2,3,6,9\}$ and $P_{6}=\{4,5,7, \underline{8}\}$.

Go to Step 7 again, and the algorithm turns out to be infeasible, so the algorithm goes to Step 11 and backtracks. The index sets are now $S_{7}^{+}=\{4,5\}$, $S_{7}^{0}=\{1,2,3,6,8,9\}$ and $P_{7}=\{4,5, \underline{7}\}$.

Go to Step 7 again, and the algorithm turns out to be infeasible, so the algorithm goes to Step 11 and backtracks. The index sets are $S_{8}^{+}=\{4\}, S_{8}^{-}=\{5\}$, $S_{8}^{0}=\{1,2,3,6,7,8,9\}$ and $P_{8}=\{4, \underline{5}\}$.

Go to Step 7, a feasible solution is found. It passes the test at Step 8 and satisfied the complementary slackness conditions at Step 9. Continuing at Step 8, $\bar{F}=-3$. The algorithm backtracks at Step 11 and updates the sets, $S_{9}^{+}=\phi, S_{9}^{-}=\{4\}, S_{9}^{0}=\{1,2,3,5,6,7,8,9\}$ and $P_{9}=\{\underline{4}\}$. Returning to Step 7, another feasible solution is found, but at Step 8, the value of the leader's objective function is greater than the incumbent upper bound, so it goes to Step 11 and backtracks. However, no live vertices exist. We have found an optimal solution, occurring at the point $\left(x^{*}, y^{*}\right)=(0,0.5),\left(u^{*}\right)=$ $(0,0,0,3,0,0,0,0,0)$ with $F^{*}=-3$ and $f^{*}=1.5$. The branch-and-bound tree is shown in Fig. 1. 


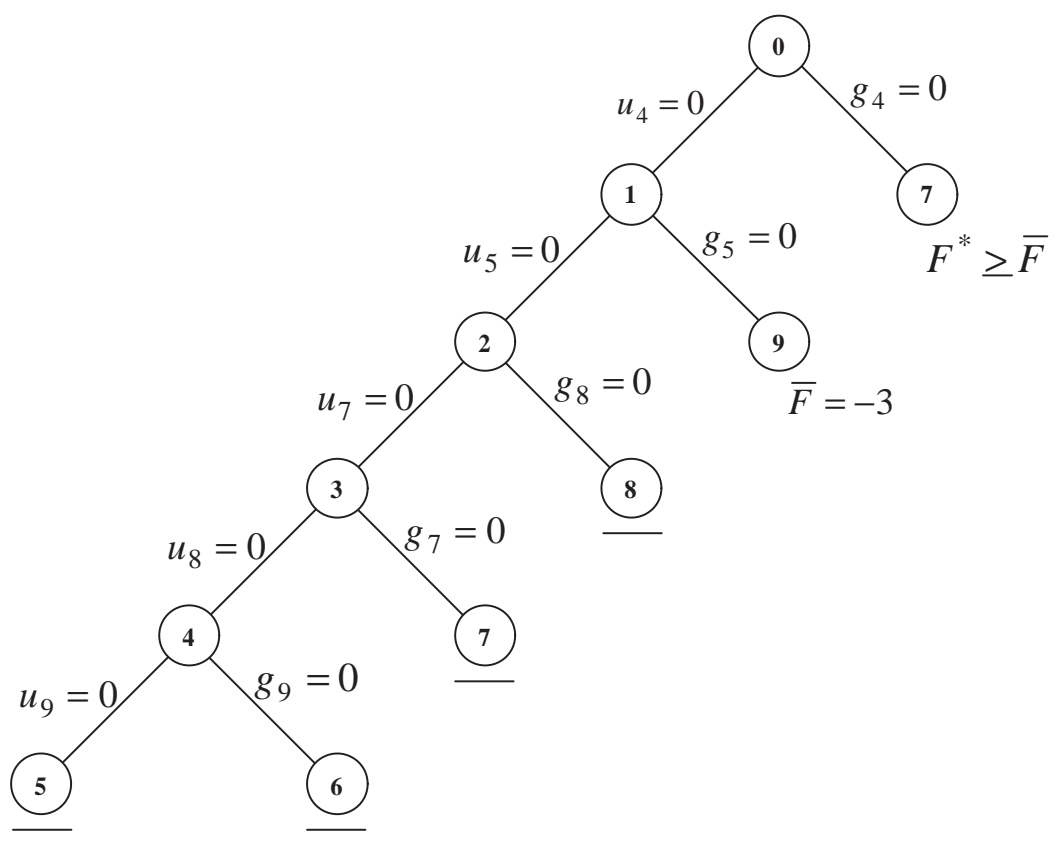

Fig. 1. Branch-and-bound search tree

By examining above procedure, we found that the optimal solution occurs at the point $\left(x^{*}, y^{*}\right)=(0,0.5)$ with

$$
\begin{aligned}
& \min _{x \in X}\left(F_{1}(x, y)\right)_{1}^{L(R)}=1 \\
& \min _{x \in X}\left(F_{1}(x, y)\right)_{0}^{L}=0.5 \\
& \min _{x \in X}\left(F_{1}(x, y)\right)_{0}^{R}=1.5 \\
& \min _{x \in X}\left(F_{2}(x, y)\right)_{1}^{L(R)}=-2 \\
& \min _{x \in X}\left(F_{2}(x, y)\right)_{0}^{L}=-2.5 \\
& \min _{x \in X}\left(F_{2}(x, y)\right)_{0}^{R}=-1.5 \\
& \min _{y \in Y}\left(f_{1}(x, y)\right)_{1}^{L(R)}=-0.5 \\
& \min _{y \in Y}\left(f_{1}(x, y)\right)_{0}^{L}=-1 \\
& \min _{y \in Y}\left(f_{1}(x, y)\right)_{0}^{R}=0 \\
& \min _{y \in Y}\left(f_{2}(x, y)\right)_{1}^{L(R)}=1 \\
& \min _{y \in Y}\left(f_{2}(x, y)\right)_{0}^{L}=0.5 \\
& \min _{y \in Y}\left(f_{2}(x, y)\right)_{0}^{R}=1.5
\end{aligned}
$$


Step 13. When $l=2$, we solve the following MOLBP problem by Step 4

$$
\begin{aligned}
& \min _{x \in X}\left(F_{1}(x, y)\right)_{1}^{L(R)}=-1 x+2 y \\
& \min _{x \in X}\left(F_{1}(x, y)\right)_{\frac{1}{2}}^{L}=-\frac{3}{2} x+\frac{3}{2} y \\
& \min _{x \in X}\left(F_{1}(x, y)\right)_{0}^{L}=-2 x+1 y \\
& \min _{x \in X}\left(F_{1}(x, y)\right)_{\frac{1}{2}}^{R}=-\frac{\sqrt{2}}{2} x+\frac{5}{2} y \\
& \min _{x \in X}\left(F_{1}(x, y)\right)_{0}^{R}=0 x+3 y \\
& \min _{x \in X}\left(F_{2}(x, y)\right)_{1}^{L(R)}=2 x-4 y \\
& \min _{x \in X}\left(F_{2}(x, y)\right)_{\frac{1}{2}}^{L}=\frac{3}{2} x-\frac{9}{2} y \\
& \min _{x \in X}\left(F_{2}(x, y)\right)_{0}^{L}=1 x-5 y \\
& \min _{x \in X}\left(F_{2}(x, y)\right)_{\frac{1}{2}}^{L}=\frac{5}{2} x-\frac{7}{2} y \\
& \min _{x \in X}\left(F_{2}(x, y)\right)_{0}^{R}=3 x-3 y \\
& \text { subject to }-1 x+3 y \leq 4 \\
& -\frac{3}{2} x+\frac{5}{2} y \leq \frac{7}{2} \\
& -2 x+2 y \leq 3 \\
& -\frac{\sqrt{2}}{2} x+\frac{7}{2} y \leq \frac{9}{2} \\
& 0 x+4 y \leq 5 \\
& \min _{y \in Y}\left(f_{1}(x, y)\right)_{1}^{L(R)}=2 x-1 y \\
& \min _{y \in Y}\left(f_{1}(x, y)\right)_{\frac{1}{2}}^{L}=\frac{3}{2} x-\frac{3}{2} y \\
& \min _{y \in Y}\left(f_{1}(x, y)\right)_{0}^{L}=1 x-2 y \\
& \min _{y \in Y}\left(f_{1}(x, y)\right)_{\frac{1}{2}}^{R}=\frac{5}{2} x-\frac{\sqrt{2}}{2} y \\
& \min _{y \in Y}\left(f_{1}(x, y)\right)_{0}^{R}=3 x-0 y \\
& \min _{y \in Y}\left(f_{2}(x, y)\right)_{1}^{L(R)}=-1 x+2 y \\
& \min _{y \in Y}\left(f_{2}(x, y)\right)_{\frac{1}{2}}^{L}=-\frac{3}{2} x+\frac{3}{2} y \\
& \min _{y \in Y}\left(f_{2}(x, y)\right)_{0}^{L}=-2 x+1 y \\
& \min _{y \in Y}\left(f_{2}(x, y)\right)_{\frac{1}{2}}^{R}=-\frac{\sqrt{2}}{2} x+\frac{5}{2} y \\
& \min _{y \in Y}\left(f_{2}(x, y)\right)_{0}^{R}=0 x+3 y
\end{aligned}
$$


subject to $1 x-1 y \leq 0$

$$
\begin{aligned}
& \frac{\sqrt{2}}{2} x-\frac{3}{2} y \leq-\frac{1}{2} \\
& 0 x-2 y \leq-1 \\
& \frac{3}{2} x-\frac{\sqrt{2}}{2} y \leq \frac{\sqrt{2}}{2} \\
& 2 x-0 y \leq 1 \\
& -\frac{3}{2} x-\frac{3}{2} y \leq-\frac{1}{2} \\
& -1 x-1 y \leq 0 \\
& -\frac{\sqrt{2}}{2} x-\frac{\sqrt{2}}{2} y \leq \frac{\sqrt{2}}{2} \\
& -2 x-2 y \leq-1 .
\end{aligned}
$$

By Step 5 to Step 12, we have

$$
\begin{aligned}
& \min _{x \in X} F(x, y)=\left(3+\frac{5-\sqrt{2}}{2}\right) x-10 y \\
& \text { subject to }-1 x+3 y \leq 4 \\
& -\frac{3}{2} x+\frac{5}{2} y \leq \frac{7}{2} \\
& -2 x+2 y \leq 3 \\
& -\frac{\sqrt{2}}{2} x+\frac{7}{2} y \leq \frac{9}{2} \\
& 4 y \leq 5 \\
& \min _{y \in Y} f(x, y)=\left(\frac{5-\sqrt{2}}{2}+3\right) x+\left(\frac{5-\sqrt{2}}{2}+3\right) y \\
& \text { subject to } 1 x-1 y \leq 0 \\
& \frac{\sqrt{2}}{2} x-\frac{3}{2} y \leq-\frac{1}{2} \\
& 0 x-2 y \leq-1 \\
& \frac{3}{2} x-\frac{\sqrt{2}}{2} y \leq \frac{\sqrt{2}}{2} \\
& 2 x-0 y \leq 1 \\
& -\frac{3}{2} x-\frac{3}{2} y \leq-\frac{1}{2} \\
& -1 x-1 y \leq 0 \\
& -\frac{\sqrt{2}}{2} x-\frac{\sqrt{2}}{2} y \leq \frac{\sqrt{2}}{2} \\
& -2 x-2 y \leq-1
\end{aligned}
$$


The optimal solution occurs at the point $\left(x^{*}, y^{*}\right)=(0,0.5)$ with

$$
\begin{aligned}
& \min _{x \in X}\left(F_{1}(x, y)\right)_{1}^{L(R)}=1 \\
& \min _{x \in X}\left(F_{1}(x, y)\right)_{\frac{1}{2}}^{L}=0.75 \\
& \min _{x \in X}\left(F_{1}(x, y)\right)_{0}^{L}=0.5 \\
& \min _{x \in X}\left(F_{1}(x, y)\right)_{\frac{1}{2}}^{R}=1.25 \\
& \min _{x \in X}\left(F_{1}(x, y)\right)_{0}^{R}=1.5 \\
& \min _{x \in X}\left(F_{2}(x, y)\right)_{1}^{L(R)}=-2 \\
& \min _{x \in X}\left(F_{2}(x, y)\right)_{\frac{1}{2}}^{L}=-2.25 \\
& \min _{x \in X}\left(F_{2}(x, y)\right)_{0}^{L}=-2.5 \\
& \min _{x \in X}\left(F_{2}(x, y)\right)_{\frac{1}{2}}^{L}=-1.75 \\
& \min _{x \in X}\left(F_{2}(x, y)\right)_{0}^{R}=-1.5 \\
& \min _{y \in Y}\left(f_{1}(x, y)\right)_{1}^{L(R)}=-0.5 \\
& \min _{y \in Y}\left(f_{1}(x, y)\right)_{\frac{1}{2}}^{L}=-0.75 \\
& \min _{y \in Y}\left(f_{1}(x, y)\right)_{0}^{L}=-1 \\
& \min _{y \in Y}\left(f_{1}(x, y)\right)_{\frac{1}{2}}^{R}=-\frac{\sqrt{2}}{4} \\
& \min _{y \in Y}\left(f_{1}(x, y)\right)_{0}^{R}=0 \\
& \min _{y \in Y}\left(f_{2}(x, y)\right)_{1}^{L(R)}=1 \\
& \min _{y \in Y}\left(f_{2}(x, y)\right)_{\frac{1}{2}}^{L}=0.75 \\
& \min _{y \in Y}\left(f_{2}(x, y)\right)_{0}^{L}=0.5 \\
& \min _{y \in Y}\left(f_{2}(x, y)\right)_{\frac{1}{2}}^{R}=1.25 \\
& \min _{y \in Y}\left(f_{2}(x, y)\right)_{0}^{R}=1.5 . \\
&
\end{aligned}
$$

Step 14. When $(x, y)=(0,0.5)$, we have $\left\|(x, y)_{2^{2}}-(x, y)_{2^{1}}\right\|=0<\varepsilon$.

Step 15. The solution of the problem is $(x, y)=(0,0.5)$ such that

$$
\begin{aligned}
& \min _{x \in X} F_{1}(x, y)=0.5 \times \tilde{2} \\
& \min _{x \in X} F_{2}(x, y)=-0.5 \times \tilde{4} \\
& \min _{y \in Y} f_{1}(x, y)=0.5 \times \tilde{2}
\end{aligned}
$$




$$
\min _{y \in Y} f_{2}(x, y)=-0.5 \times \tilde{1}
$$

This example shows how the approximation branch-and-bound algorithm is used to solve a FMOLBP problem. We now present another example for explain now to build a FMOLBP model.

Example 23 In a company, the CEO is as the leader, and the heads of branches of the company are as the follower in making an annual budget for the company. Obviously, the leader (the CEO)'s decision will be affected by the reactions of the follower (heads of branches). Each of the CEO's possible decisions is influenced by the various reactions of the heads. In order to arrive an optimal solution (better strategies) for the CEO's decision on the annual budget, we establish a bilevel decision making model.

The CEO has two main objectives: 1) to maximize the net profits, represented by $F_{1}(x, y)$ and 2$)$ to maximize the quality of products, by $F_{2}(x, y)$ but subject to some constraints including the requirements of material, marking cost, labor cost, working hours and so on. The heads of branches, as the follower, attempts to 1) maximize their net profit, $f_{1}(x, y)$, and 2) maximize work satisfactory $f_{2}(x, y)$ The CEO understands that for each policy he may make, these heads will have a new reaction to deal with by optimizing their objective $\max _{y \in Y}\left(f_{1}(x, y), f_{2}(x, y)\right)$.

When modeling the bilevel decision problem, the main difficulty is to set up parameters for the objectives and constraints of both the leader and the follower. We can only estimate some values such as material cost, labor cost, according to our experience and previous data. For some items, the values can be only assigned by linguistic terms, such as 'about $\$ 1000$ '. This is a common case in any organizational decision practice. By using fuzzy numbers to describe these uncertain values and linguistic terms in parameters, a FMOLBP model can be established for this decision problem.

Let $x=\left(x_{1}, x_{2}\right)^{T} \in \mathbb{R}^{2}$ be the CEO's decision variables, and $y=\left(x_{1}, x_{2}, x_{3}\right)^{T} \in$ $\mathbb{R}^{3}$ be the branch heads' decision variables, and $X=\{x \geqslant 0\}, Y=\{y \geqslant 0\}$, we can build the following model for the decision problem:

$$
\begin{gathered}
\max _{x \in X} F_{1}(x, y)=(\tilde{1}, \tilde{9})\left(x_{1}, x_{2}\right)^{T}+(\widetilde{10}, \tilde{1}, \tilde{3})\left(y_{1}, y_{2}, y_{3}\right)^{T} \\
\max _{x \in X} F_{2}(x, y)=(\tilde{9}, \tilde{2})\left(x_{1}, x_{2}\right)^{T}+(\tilde{2}, \tilde{7}, \tilde{4})\left(y_{1}, y_{2}, y_{3}\right)^{T} \\
\text { subject to }(\tilde{3}, \tilde{9})\left(x_{1}, x_{2}\right)^{T}+(\tilde{9}, \tilde{5}, \tilde{3})\left(y_{1}, y_{2}, y_{3}\right)^{T} \leqslant 10 \tilde{3} 9 \\
\quad(-\widetilde{4},-\widetilde{1})\left(x_{1}, x_{2}\right)^{T}+(\tilde{3},-\widetilde{3}, \tilde{2})\left(y_{1}, y_{2}, y_{3}\right)^{T} \leqslant \tilde{94} \\
\max _{y \in Y} f_{1}(x, y)=(\tilde{4}, \tilde{6})\left(x_{1}, x_{2}\right)^{T}+(\tilde{7}, \tilde{4}, \tilde{8})\left(y_{1}, y_{2}, y_{3}\right)^{T} \\
\max _{y \in Y} f_{2}(x, y)=(\tilde{6}, \tilde{4})\left(x_{1}, x_{2}\right)^{T}+(\tilde{8}, \tilde{7}, \tilde{4})\left(y_{1}, y_{2}, y_{3}\right)^{T} \\
\quad \text { subject to }(\tilde{3},-\widetilde{9})\left(x_{1}, x_{2}\right)^{T}+(-\tilde{9},-\tilde{4}, \tilde{0})\left(y_{1}, y_{2}, y_{3}\right)^{T} \leqslant \widetilde{61}
\end{gathered}
$$




$$
\begin{aligned}
& (\tilde{5}, \tilde{9})\left(x_{1}, x_{2}\right)^{T}+(\widetilde{10},-\widetilde{1},-\widetilde{2})\left(y_{1}, y_{2}, y_{3}\right)^{T} \leqslant \widetilde{924} \\
& (\tilde{3},-\widetilde{3})\left(x_{1}, x_{2}\right)^{T}+(\tilde{0}, \tilde{1}, \tilde{5})\left(y_{1}, y_{2}, y_{3}\right)^{T} \leqslant \widetilde{420}
\end{aligned}
$$

In this model, the unified form for all membership functions of the parameters of the objective functions and constraints is as follows:

$$
\mu_{\tilde{\alpha}}(x)= \begin{cases}0, & x<a \text { or } c<x, \\ \left(x^{2}-a^{2}\right) /\left(b^{2}-a^{2}\right) & , a \leqslant x<b, \\ 1, & x=b, \\ \left(c^{2}-x^{2}\right) /\left(c^{2}-d^{2}\right), & b<x \leqslant c .\end{cases}
$$

For simplicity, we only represent the above form of membership function as (a, b, c). Then, for the example, all membership functions of fuzzy parameters of the objective functions and constraints are to be represented in the quadruple pair form and listed in Tables 2,3 , and 4, respectively.

Table 2

Membership functions of fuzzy objective functions' parameters

\begin{tabular}{c|ccccc}
\hline$\tilde{c}_{i j}$ & 1 & 2 & 3 & 4 & 5 \\
\hline 1 & $(0,1,2)$ & $(8,9,12)$ & $(9,10,13)$ & $(0.5,1,2.5)$ & $(2,3,6)$ \\
\hline 2 & $(8,9,12)$ & $(1,2,5)$ & $(1,2,5)$ & $(6,7,10)$ & $(3,4,7)$ \\
\hline 3 & $(2,4,5)$ & $(4,6,7)$ & $(5,7,8)$ & $(2,4,5)$ & $(6,8,9)$ \\
\hline & $(4,6,7)$ & $(2,4,5)$ & $(6,8,9)$ & $(5,7,8)$ & $(2,4,5)$ \\
\hline
\end{tabular}

Table 3

Membership functions of fuzzy constraints' parameters

\begin{tabular}{c|ccccc}
\hline$a_{i j}$ & 1 & 2 & 3 & 4 & 5 \\
\hline 1 & $(2,3,5)$ & $(8,9,11)$ & $(8,9,11)$ & $(4,5,7)$ & $(2,3,5)$ \\
\hline 2 & $(-6,-4,-3)$ & $(-2,-1,-0.5)$ & $(2,3,5)$ & $(-5,-3,-2)$ & $(-4,-2,-1)$ \\
\hline 3 & $(2,3,5)$ & $(-11,-9,-8)$ & $(-11,-9,-8)$ & $(-6,-4,-3)$ & $(0,0,0)$ \\
\hline 4 & $(4,5,7)$ & $(8,9,11)$ & $(9,10,12)$ & $(0.5,1,2)$ & $(-4,-2,-1)$ \\
\hline 5 & $(2,3,5)$ & $(-5,-3,-2)$ & $(0,0,0)$ & $(0.5,1,2)$ & $(4,5,7)$ \\
\hline
\end{tabular}

Now, We first given the weights for the two fuzzy objectives of the leader are $(0.5,0.5)$ and of the follower $(0.5,0.5)$ and the interval $[0,1]$ be decomposed into $2^{l-1}$ equal sub-intervals with $\left(2^{l-1}+1\right)$ nodes $\lambda_{i}\left(i=0, \ldots, 2^{l-1}\right)$ which is arranged in the order of $0=\lambda_{0}<\lambda_{1}<\cdots<\lambda_{2^{l-1}}=1$ and a range of errors $\epsilon=10^{-6}>0$. Then we can solve this problem by using the proposed approximation branch-and-bound approach. The solution of the problem is 
Table 4

Membership functions of fuzzy right-hand-side's parameters

\begin{tabular}{c|c}
\hline$\tilde{b}$ & 1 \\
\hline 1 & $(1038,1039,1041)$ \\
\hline 2 & $(93,94,96)$ \\
\hline 3 & $(60,61,63)$ \\
\hline 4 & $(923,924,926)$ \\
\hline 5 & $(419,420,422)$ \\
\hline
\end{tabular}

$x_{1}=146.2955, x_{2}=28.9394$ and $y_{1}=0, y_{2}=67.9318, y_{3}=0$ such that

$$
\begin{aligned}
& \max _{x \in X} F_{1}(x, y)=164.2955 \times \tilde{1}+28.9394 \times \tilde{9}+67.9318 \times \tilde{1} \\
& \max _{x \in X} F_{2}(x, y)=164.2955 \times \tilde{9}+28.9394 \times \tilde{2}+67.9318 \times \tilde{7} \\
& \max _{y \in Y} f_{1}(x, y)=164.2955 \times \tilde{4}+28.9394 \times \tilde{6}+67.9318 \times \tilde{4} \\
& \max _{y \in Y} f_{2}(x, y)=164.2955 \times \tilde{6}+28.9394 \times \tilde{4}+67.9318 \times \tilde{7}
\end{aligned}
$$

This problem can be solved by using the proposed approximation branch-andbound algorithm. Based on the algorithm, a bilevel decision support system has been developed for helping get a solution effectively. Fig. 2 shows the process of the solution.

\section{Conclusions and Further Study}

A bilevel decision problem may have multiple objective functions and fuzzy parameters can appear in both the objectives and constraints of the leader and the follower. The main research issue is how to derive an optimal solution for such a FMOLBP problem. This paper proposes a fuzzy number based approximation branch-and-bound algorithm to this issue. A cased based example and a numeral example are then given to illustrate the proposed FMOLBP model and the approximation branch-and-bound algorithm. Further study on this study includes the development of models and approaches for fuzzy bilevel multi-follower programming problems. In such a kind of problems, multiple followers are involved and the leader's decision will be affected not only by those followers' individual reactions but also by the relationships among these followers. As uncertain information could occur in the objectives and the constraints of both the leader and his/her multiple followers, one of the challenges is how to get an optimal solution for the leader in the complex environment. A model-driven decision support system will be developed for implementing the proposed approaches. 


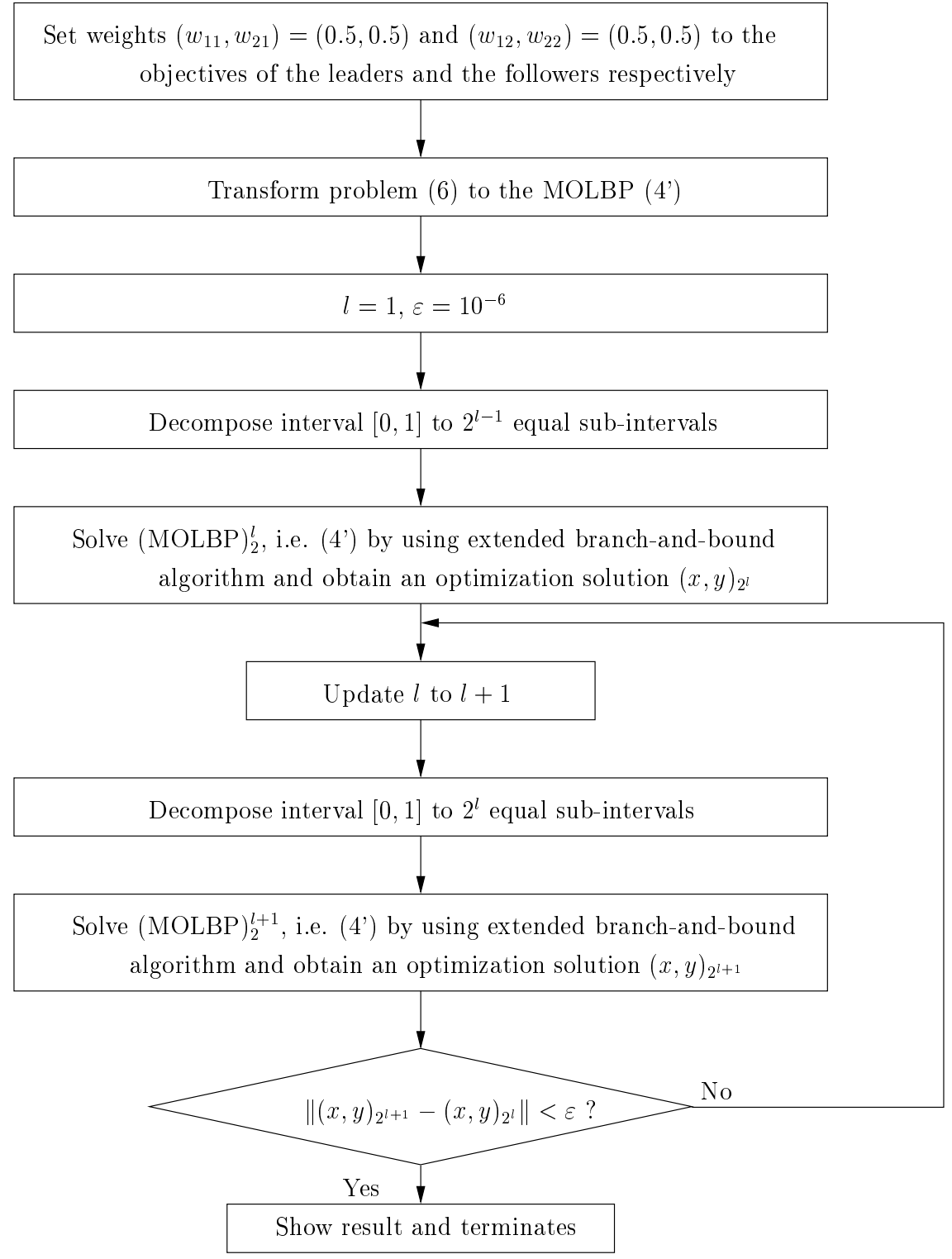

Fig. 2. A flowchart of the approximation branch-and-bound algorithm

\section{Acknowledgments}

The work presented in this paper was supported by Australian Research Council (ARC) under discovery grants DP0557154.

\section{References}

[1] G. Anandalingam and T. Friesz, Hierarchical optimization: An introduction, Annals of Operations Research 34(1992) 1-11

[2] J. Bard, Practical bilevel optimization: Algorithms and applications, Kluwer 
Academic Publishers, Amsterdam, 1998

[3] J. Bard and J. Falk, An explicit solution to the programming problem, Computers and Operations Research 9(1982) 77-100

[4] W. Bialas and M. Karwan, Two-level linear programming, Management Science 30(1984) 1004-1020

[5] J. Bracken and J. McGill, Mathematical programs with optimization problems in the constraints, Operations Research 21(1973) 37-44.

[6] W. Candler and R. Townsley, A linear two-level programming problem, Computers and Operations Research 9(1982) 59-76

[7] Y. Chen, M. Florian and S. Wu, A descent dual approach for linear bilevel programs. Technical Report CRT-866, Centre de Recherche sur les Transports 1992

[8] S. Dempe, A simple algorithm for the linear bilevel programming problem, Optimization 18(1987) 373-385

[9] C. L. Hwang and A. S. M. Masud, Multiple objective decision-making - Methods and applications: A state of the art survey, Springer, New York, 1979

[10] P. Hansen, B. Jaumard, and G. Savard, New branch-and-bound rules for linear bilevel programming. SIAM Journal on Scientific and Statistical Computing 13(1992) 1194-1217

[11] J. Lu, C. Shi, and G. Zhang, An extended branch and bound algorithm for bilevel multi-follower decision making in a referential-uncooperative situation, accepted by International Journal of Information Technology And Decision Making 2006

[12] T. Miller, T. Friesz and R. Tobin, Heuristic algorithms for delivered price spatially competitive network facility location problems, Annals of Operations Research 34(1992) 177-202

[13] M. Sakawa, Fussy sets and interactive mulitobjective optimization, Plenum Press, New York, 1993

[14] M. Sakawa, I. Nishizaki, and Y. Uemura, Interactive fuzzy programming for multilevel linear programming problems with fuzzy parameters, Fuzzy Sets and Systems 109(2000) 3-19

[15] M. Sakawa, I. Nishizaki, and Y. Uemura, Interactive fuzzy programming for multilevel linear programming problems with fuzzy parameters, Fuzzy Sets and Systems 109(1)(2000) 3-19

[16] M. Sakawa, I. Nishizaki and Y. Uemura (2000), Interactive fuzzy programming for two-level linear fractional programming problems with fuzzy parameters, Fuzzy Sets and Systems 115(1)(2000) 93-103 
[17] M. Sakawa and K. Yauchi, Interactive decision making for multiobjective nonconvex programming problems with fuzzy numbers through coevolutionary genetic algorithms, Fuzzy Sets and Systems 114(1)(2000) 151-165

[18] M. Sakawa and I. Nishizaki, Interactive fuzzy programming for two-level linear fractional programming problems, Fuzzy Sets and Systems 119(1)(2001) 31-40

[19] M. Sakawa and I. Nishizaki, Interactive fuzzy programming for decentralized two-level linear programming problems, Fuzzy Sets and Systems 125(3)(2001) 301-315

[20] M. Sakawa and I. Nishizaki, Interactive fuzzy programming for two-level nonconvex programming problems with fuzzy parameters through genetic algorithms, Fuzzy Sets and Systems 127(2)(2002) 185-197

[21] M. Sakawa and I. Nishizaki, Interactive fuzzy programming for two-level conconvex programming problems with fuzzy parameters through genertic algorithms, Fuzzy Sets and Systems 127 (2002) 185-197

[22] C. Shi, J. Lu and G. Zhang, An extended Kuhn-Tucker approach for linear bilevel programming, Applied Mathematics and Computation 162(2005) 51-63

[23] C. Shi, J. Lu, G. Zhang and H. Zhou, An extended branch and bound algorithm for linear bilevel programming, Applied Mathematics and Computation 180(2006) 529-537

[24] H. Shih and E. Lee, Compensatory fuzzy multiple level decision making, Fuzzy Sets and Systems 114(2000) 71-87

[25] S. Sinha, Fuzzy programming approach to multi-level programming problems, Fuzzy Sets and Systems 136(2003) 189-202

[26] F. Tiryaki, Interactive compensatory fuzzy programming for decentralized multi-level linear programming (DMILP) problems, Fuzzy Sets and Systems 157(2006) 3072-3090

[27] D. White and G. Anandalingam, A penalty function approach for solving bilevel linear programs, Journal of Global Optimization 3(1993) 397-419

[28] L. A Zadeh, Fuzzy sets, Information $\& 5$ Control 8(1965) 338-353.

[29] G. Zhang and J. Lu, The definition of optimal solution and an extended KuhnTucker approach for fuzzy linear bilevel programming, The IEEE Computational Intelligence Bulletin 5(2005) 1-7

[30] G. Zhang and J. Lu, Model and approach of fuzzy bilevel decision making for logistics planning problem, Accepted by Journal of Enterprise Information Management, 2005

[31] G. Zhang, J. Lu and T. Dillon, A branch-and-bound algorithm for fuzzy bilevel decision making, in: Ruan, D'hondt, Fantoni, De Cock, Nachtegael and Kerre, eds., Applied Artificial Intelligence, World Scientific, Singapore, 2006 291-298 
[32] G. Zhang, J. Lu and T. Dillon, An approximation Kuhn-Tucker approach for fuzzy linear bilevel decision making problems, submitted to book "Intelligent Decision Making" (Spinger), edited by Gloria Wren and Lakhmi Jain, 2006

[33] G. Zhang, J. Lu and T. Dillon, Solution concepts and an approximation KuhnTucker approach for fuzzy multi-objective linear bilevel programming, accepted by book "Pareto Optimality, Game Theory and Equilibria" (Spinger), edited by Panos Pardalos and Altannar Chinchuluun, 2006 